\title{
ELECTROMAGNETIC SCATTERING FROM \\ CYLINDRICALLY AND SPHERICALLY STRATIFIED BODIES
}

Thesis by

Raymond W. Latham

In Partial Fulfillment of the Requirements

For the Degree of

Doctor of Philosophy

California Institute of Technology

Pasadena, California

1968

(Submitted September 29, 1967) 


\section{ABSTRACT}

A method is developed for calculating the electromagnetic fiela scattered by certain types of bodies. The bodies consist of inhomogeneous media whose constitutive parameters vary only with the aistance from some axis or point of symmetry. The method consists in an extension of the invariant imbedding method for treating wave problems. This method, which is familiar in the case of a onedimensional inhomogeneity, is extended to handle special types of two and three-dimensional inhomogeneities. Comparisons are made with other methods which have been proposed for treating these kinds of problems. Examples of applications of the method are given, some of which are of interest in themselves. 
The author is grateful to Professor C. H. Papas for his guidance and encouragement during the course of this work.

Thanks are also due to Mrs. R. Stratton for typing the text, and to Dr. K. S. H. Lee for his suggestions and criticisms. 


$$
-i v-
$$

TABLE OF CONTENTS

1. INTRODUCTION

2. BACKGROUND 6

A. Separation of Variables 6

B. Thin Shell Approximation 16

C. Invariant Imbedding in Plane Stratified Media 21

3. REFLECTION TREATMENT OF CYLINDRICALLY AND SPHERICALLY $\begin{array}{ll}\text { STRATIFIED MEDIA } & 37\end{array}$

4. SURVEY AND COMPARISONS 61

5. EXAMPLES OF CALCULATIONS FOR THE CYLINDRICAL CASE 66

6. CONCLUDING REMARKS 81

APPENDIX: COMPUTER CODE 85

REFERENCES 90 


\section{INTRODUCTION}

This report is an exposition of a method which has been developed for treating the effect on plane, time-harmonic, electromagnetic waves of two particular types of inhomogeneous media. The two types consist of media in which the constitutive parameters, namely the dielectric constant $\varepsilon$ and the permeability $\mu$ vary, in one case only with the distance from an axis of symmetry, and in the other case only with the distance from a point of symmetry. It is assumed that the constitutive parameters are scalars. It is also assumed that these medium parameters become constant at a finite distance from the axis or point of symmetry. This last assumption makes it meaningful to treat the problem as a "scattering problem" in the usual sense, the total field in the constant parameter region being made up of an incident wave of simple form, and a scattered wave, which is produced by the inhomogeneity and is to be calculated. In particular we are primarily interested in the form of the scattered wave a great distance from the scattering region.

When, in the future, we wish to refer to the first configuration described above, we will speak of the cylindrical case. In this case we will take as our incident wave one whose propagation vector is perpendicular to the axis of symmetry. Two independent polarizations of the incident wave, namely those with electric and magnetic vector parallel to the axis, will be considered. In the spherical case, of course, there is really only one polarization.

Even with the large amount of symmetry which is assumed in the scattering region, the inhomogeneous nature of the problem makes a 
certain amount of numerical computation inevitable except for special cases where the variation of the constitutive parameters with radius is a function of very simple form. The method which we put forth is based on an attempt to incorporate as much theory as possible into the formulation in order to minimize this numerical work. Formulas are aeveloped by which only the reflection coefficients of the cylinarical or spherical modes are calculated. This statement will be explained in detail later, but the point here is that many needless computations are avoided by concentrating on the scattered field alone. In particular, evaluation of the field at each point within the inhomogeneity is bypassea. This calculation of only the reflection coefficients is sufficient since in experimental and practical situations the only quantities of interest are the scattered fields at a great distance from the scatterer.

The formulation arrived at bears a certain resemblance to others which have been used in the past for similar problems. Because of the desirability of a complete discussion of the present method, the second section contains a summary of work which has been done previously along similar lines, for purposes of comparison later on. This section also introduces (in their simplest form) some ideas which are used later.

In particular, Section $2 \mathrm{~A}$ treats the method of separation of variables as it applies to our special cases of symmetry. This serves partly as a detailed introduction to the problem and its points of difficulty. It also provides a fair amount of completeness, and the data which may be obtained for certain simple constitutive parameter functions based on calculations using this method, are valuable in checking results obtained by the method proposed in this report. 
Section $2 B$ deals with another previous way of treating our symmetrical inhomogeneities. This is a straightforward method of dividing the cylinder or sphere up into many thin shells in which the constitutive parameters are assumed constant. For each angular mode a radial wave function of general form, involving two arbitrary constants, is assumed in each shell and the boundary conditions on the electric and magnetic fields are invoked at each shell boundary. This procedure results in a large set of equations involving the constants multiplying the wave functions in all the layers. One reason this method is mentioned is because one of the lines of development of the method which is proposed in this report starts with the concept of a thin uniform shell within which there are two independent wave functions for each angular mode. Another reason for the inclusion of this subsection is to demonstrate the reduction in necessary calculations when the problem is reformulated so that only desired quantities are computed.

Subsection $2 \mathrm{C}$ is the most important of Section 2. It treats the method of invariant imbedding as it applies to the calculation of scattering from a medium which has constitutive parameters varying along only one axis of a three-dimensional Cartesian system. Thus we are able to explore the ideas and details of the method in this, the simplest problem in wave propagation to which it may profitably be applied. The method of the present report is essentially that of part $2 \mathrm{C}$ extended to cylindrical and spherical geometries with some modifications for more convenient calculation in these special symmetries. 
The third section constitutes the main work of the report. As far as the algebra goes, we concentrate mostly on the cylindrical case. The point of this is to keep the ideas as unobscured as possible by a mass of formulas, the cylinder being better than the sphere for this purpose. The necessary extra discussion for the spherical case is given, including the final equations, but the bulk of the algebra for the spherical case is omitted. In spite of this the remaining formulas, even in their final forms, are rather bulky. Their justification lies, not in their mathematical elegance or their transparent physical interpretation, neither of which they possess, but in the relatively small amount of additional calculation necessary for any particular case fitting one of the geometries treated. There are many objects with these types of symmetry which are currently of interest, both theoretically and practically.

In Section 4 the work of the preceding sections is combined. Comparisons of the proposed method are made with previous work both as an improvement on past methods of treating cylinder and sphere problems, and as an extension of preceding work on invariant imbedaing in the inhomogeneous one-dimensional case. Again it is emphasized that the method of invariant imbedding reduces the calculations necessary in a problem by restricting attention to only those quantities which are of interest in a practical situation.

The fifth section consists of a number of applications and examples of the methods developed. Calculations are checked with work published recently on scattering from cylindrically symmetric inhomogeneities. Another comparison is with what may be termed a cylindrical 
Luneberg lens which has been the subject of a study by H. Jasik. Further comparisons are made with scattering calculations for inhomogenieties simple enough for their reflection coefficients to be readily evaluated. Other examples are given for which no comparisons may be easily made, simply to demonstrate the wide applicability of the method and in the hope that they may be of use to those engaged in similar stuãies.

In the concluding remarks a general evaluation of the method as a practical tool is made. Also a short summary is made of certain points of secondary but interesting nature which present themselves or are used as examples throughout the analysis. In addition, a discussion is given of the possibilities for the extension of the methods to other problems not previously discussed. 


\section{$-6-$ \\ Section 2 \\ BACKGROUND}

\section{A. Separation of Variables}

The specific scattering problems which are dealt with in this report may be treated by the method of separation of variables. There is a discussion in Section 4 of some of the difficulties inherent in too straightforward an application of this method. However, a formulation of our problems in this manner will prove useful as a detailed introduction to the problems and also for the very purpose of later comparisons.

Figure 1 is a picture of the coordinate system which will be used throughout for the cylindrical case. The axis of symmetry of the medium parameters will be taken to be the $z$ axis so that $\varepsilon$ and $\mu$ are functions of $\rho$ only. The entire inhomogeneity of the medium is contained within the space $\rho<\rho_{c}$ for some $\rho_{c}$. For $\rho>\rho_{c}, \varepsilon$ and $\mu$ are independent of position and will be denoted $\varepsilon_{c}$ and $\mu_{c}$. The incident waves are assumed to travel in the positive $\mathrm{x}$ direction $(\varnothing=0$ direction $)$. For the $E$ polarization problem the only components

which the incident fields possess are $\mathrm{E}_{\mathrm{z}}^{\mathrm{inc}}$ and $\mathrm{H}_{\mathrm{y}}^{\text {inc }}$ (Figure IA). Similarly for the $H$ polarization problem the only components which the incident fields possess are $\mathrm{H}_{\mathrm{z}}^{\text {inc }}$ and $\mathrm{E}_{\mathrm{y}}^{\text {inc }}$ (Figure IB). From the solutions to problems with these two polarizations, the solution to the problem with an incident wave propagating perpendicular to the axis of symmetry and with arbitrary polarization may be determined. 
A
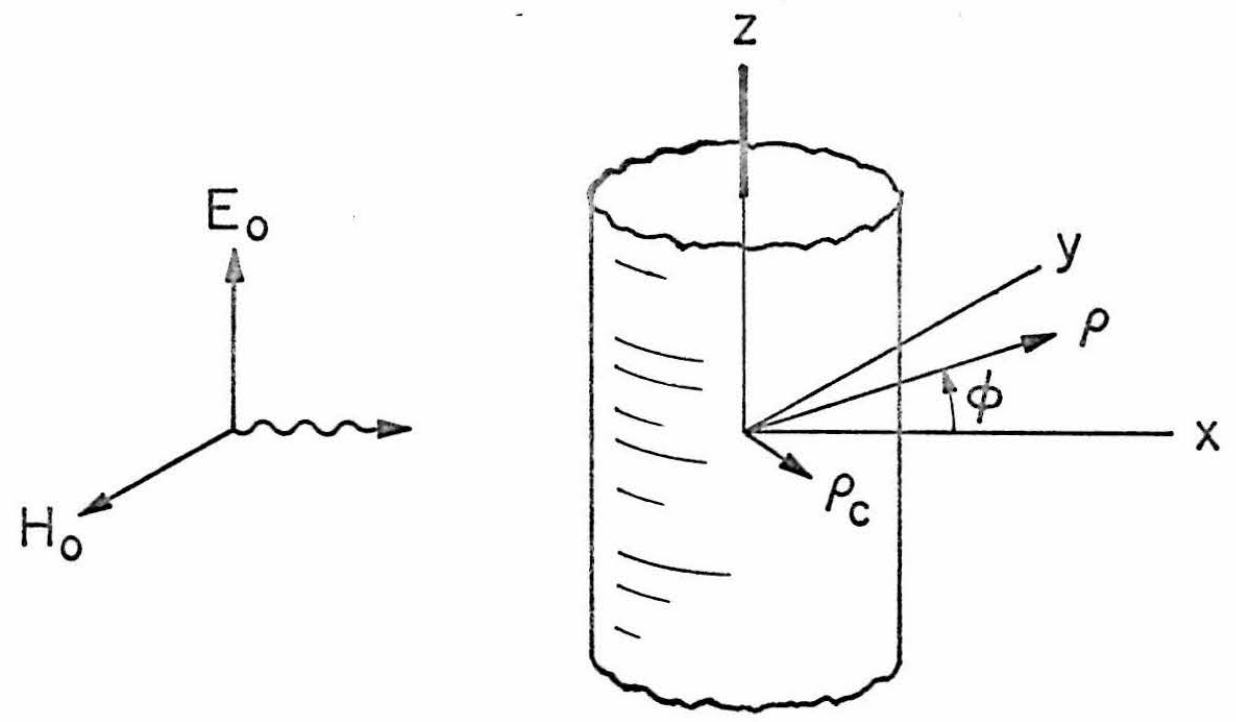

$B$
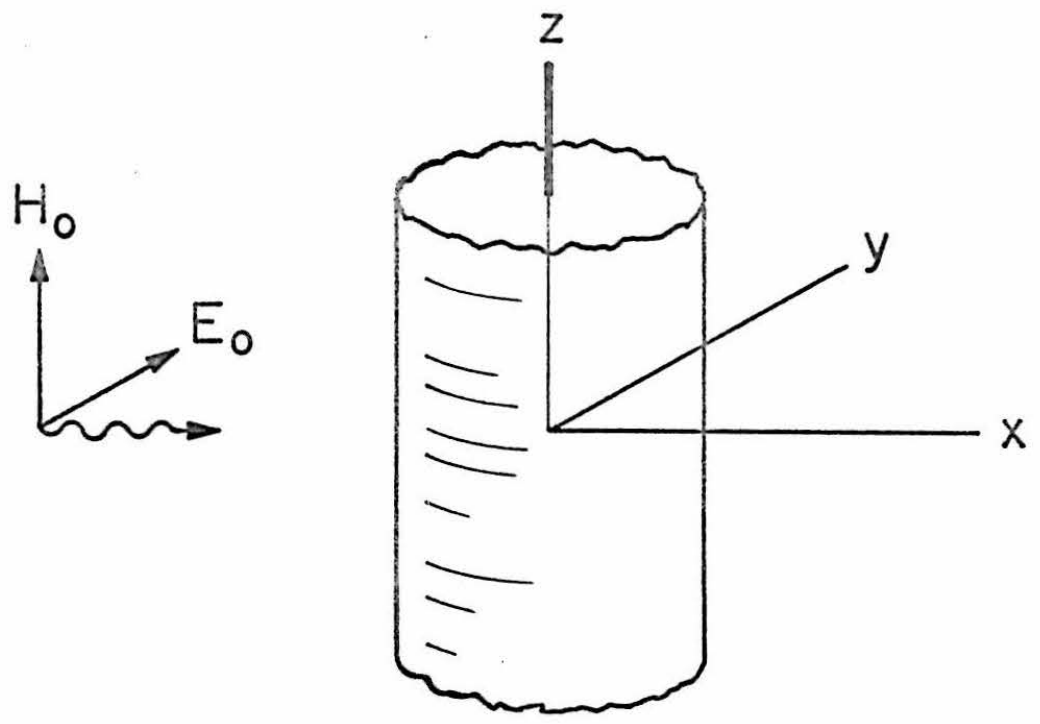

Fig. 1. The two basic problems for an axially symmetric inhomogeneity 
The derivation of the equations required by the separation of variables method for the cylinarical case will start directly from Niaxwell's equations for an assumed harmonic time dependence. That is to say, assuming

$$
\begin{aligned}
& \underline{E}(\underline{r}, t)=\operatorname{Re} \underline{E}(\underline{r}) e^{-i \omega t} \\
& \underline{B}(\underline{r}, t)=\operatorname{Re} \underline{B}(\underline{r}) e^{-i \omega t} \\
& \underline{D}(\underline{r}, t)=\operatorname{Re} \underline{D}(\underline{r}) e^{-i \omega t} \\
& \underline{H}(\underline{r}, t)=\operatorname{Re} \underline{H}(\underline{r}) e^{-i \omega t}
\end{aligned}
$$

Maxwell's equations, with no source become:

$$
\begin{gathered}
\nabla \times \underline{E}(\underline{r})=i \omega \underline{B}(\underline{r}) \\
\nabla \times \underline{H}(\underline{r})=-i \omega \underline{D}(\underline{r}) \\
\nabla \cdot \underline{D}(\underline{r})=0 \\
\nabla \cdot \underline{B}(\underline{r})=0
\end{gathered}
$$

The medium is assumed to be one for which a scalar dielectric constant and permeability constant may be defined, although these "constants" may be functions of position. Using this assumption

$$
\begin{aligned}
& \underline{D}(\underline{r})=\varepsilon(\underline{r}) \underline{E}(\underline{r}) \\
& \underline{B}(\underline{r})=\mu(\underline{r}) \underline{H}(\underline{r})
\end{aligned}
$$

Using $2.7,2.8,2.9,2.10$

$$
\nabla \cdot \varepsilon(\underline{r}) \underline{E}(\underline{r})=\nabla \cdot \mu(\underline{r}) \underline{H}(\underline{r})=0
$$

hence, 


$$
\begin{gathered}
\nabla \cdot \underline{E}(\underline{r})=-\frac{\nabla \varepsilon(\underline{r}) \cdot \underline{E}(\underline{r})}{\varepsilon(\underline{r})} \\
\nabla \cdot \underline{H}(\underline{r})=-\frac{\nabla \mu(\underline{r}) \cdot \underline{H}(\underline{r})}{\mu(\underline{r})} .
\end{gathered}
$$

Also, taking the curls of 2.5 and 2.6 and using 2.9 and 2.10

$$
\begin{aligned}
& \nabla \times \nabla \times \underline{E}(\underline{r})=i \omega \nabla \times[\mu(\underline{r}) \underline{H}(\underline{r})] \\
& \nabla \times \nabla \times \underline{H}(\underline{r})=-i \omega \nabla \times[\varepsilon(\underline{r}) \underline{E}(\underline{r})]
\end{aligned}
$$

which, using vector identities may be written

$$
\begin{aligned}
& \nabla \nabla \cdot \underline{E}-\nabla^{2} \underline{E}=i \omega[\nabla \mu \times \underline{H}+\mu \nabla \times \underline{H}] \\
& \nabla \nabla \cdot \underline{H}-\nabla^{2} \underline{H}=-i \omega[\nabla \varepsilon \times \underline{E}+\varepsilon \nabla \times \underline{E}]
\end{aligned}
$$

Again, employing $2.5,2.6,2.12$ and 2.13 , the above equations may be put in the form

$$
\begin{aligned}
& -\nabla\left(\frac{\nabla \varepsilon}{\varepsilon} \cdot \underline{E}\right)-\nabla^{2} \underline{E}=i \omega\left[\frac{\nabla \mu \times(\nabla \times \underline{E})}{i \omega \mu}-i \omega \mu \varepsilon \underline{E}\right] \\
& -\nabla\left(\frac{\nabla \mu}{\mu} \cdot \underline{\underline{H}}\right)-\nabla^{2} \underline{H}=-i \omega\left[\frac{\nabla \varepsilon \times(\nabla \times \underline{H})}{-i \omega \varepsilon}+i \omega \mu \varepsilon \underline{H}\right]
\end{aligned}
$$

Defining $k^{2}(\underline{r})=\omega^{2} \mu(\underline{r}) \varepsilon(\underline{r})$ and using further vector identitities, the above equations may be put in the following form, which is useful for further calculation. 


$$
\begin{aligned}
& {\left[\nabla^{2}+k^{2}(\underline{r})\right] \underline{E}(\underline{r})-\left(\frac{\nabla \mu}{\mu} \cdot \nabla\right) \underline{E}=(\underline{E} \cdot \nabla) \frac{\nabla \mu}{\mu}-\nabla\left\{\frac{\nabla \varepsilon}{\varepsilon} \cdot \underline{E}+\frac{\nabla \mu}{\mu} \cdot \underline{E}\right\}} \\
& {\left[\nabla^{2}+k^{2}(\underline{r})\right] \underline{H}(\underline{r})-\left(\frac{\nabla \varepsilon}{\varepsilon} \cdot \nabla\right) \underline{H}=(\underline{H} \cdot \nabla) \frac{\nabla \varepsilon}{\varepsilon}-\nabla\left\{\frac{\nabla \mu}{\mu} \cdot \underline{H}+\frac{\nabla \varepsilon}{\varepsilon} \cdot \underline{H}\right\}}
\end{aligned}
$$

Now in the cylindrical case with $E$ polarization, $\underline{E} \cdot \nabla \mu=\underline{E} \cdot \nabla \varepsilon=0$ and the $\mathrm{z}$ component of equation 2.14 , since $\mathrm{E}_{\mathrm{z}}$ does not vary with $\mathrm{z}$, becomes

$$
\left[\nabla_{2}^{2}+k^{2}(\underline{r})\right] E_{z}(\underline{\rho})-\left[\frac{\nabla_{2} \mu(\underline{\rho})}{\mu(\underline{\rho})} \cdot \nabla_{2}\right] E_{z}(\underline{\rho})=0
$$

where $\nabla_{2}=e_{x} \frac{\partial}{\partial x}+e_{y} \frac{\partial}{\partial y}$

Similarly for $H$ polarization in the cylindrical case equation

\subsection{5 becomes}

$$
\left[\nabla_{2}^{2}+k^{2}(\underline{r})\right] H_{z}(\underline{\rho})-\left[\frac{\nabla_{2} \varepsilon(\underline{\rho})}{\varepsilon(\underline{\rho})} \cdot \nabla_{2}\right] H_{z}(\underline{\rho})=0
$$

For the cylindrical geometries considered $\nabla_{2}^{\mu}$ and $\nabla_{2} \varepsilon$ have only radial components so with the use of the $\rho, \varnothing, z$ coordinate system diagrammed in Figure $1,2.16$ and 2.17 may be written more explicitly as

$$
\begin{array}{r}
\frac{I}{\rho} \frac{\partial}{\partial \rho}\left(\rho \frac{\partial E_{z}(\rho, \phi)}{\partial \rho}\right)+\frac{1}{\rho^{2}} \frac{\partial^{2} E_{z}(\rho, \phi)}{\partial \phi^{2}}-\frac{1}{\mu(\rho)} \frac{d \mu(\rho)}{d \rho} \frac{\partial E_{z}(\rho, \phi)}{\partial \rho} \\
+k^{2}(\rho) E_{z}(\rho, \phi)=0
\end{array}
$$

and 


$$
\begin{array}{r}
\frac{I}{\rho} \frac{\partial}{\partial \rho}\left(\rho \frac{\partial \mathrm{H}_{\mathrm{z}}(\rho, \phi)}{\partial \rho}\right)+\frac{1}{\rho^{2}} \frac{\partial^{2} \mathrm{H}_{\mathrm{z}}(\rho, \phi)}{\partial \phi^{2}}-\frac{1}{\varepsilon(\rho)} \frac{\mathrm{d} \varepsilon(\rho)}{\mathrm{d} \rho} \frac{\partial \mathrm{H}_{\mathrm{z}}(\rho, \phi)}{\partial \rho}+ \\
+\mathrm{k}^{2}(\rho) \mathrm{H}_{\mathrm{z}}(\rho, \phi)=0
\end{array}
$$

for the $E$ and $H$ polarization cases respectively. Hereafter the $z$ subscript on $\mathrm{E}$ and $\mathrm{H}$ will be omitted.

Since $\varnothing$ is a physical space variable $E$ and $H$ are periodic in $\varnothing$. This justifies an assumption of Fourier series expansions of $E$ and $H$ in the form

$$
\begin{aligned}
& E(\rho, \phi)=\sum_{m=-\infty}^{\infty} E_{m}(\rho) e^{i m \phi} \\
& H(\rho, \varnothing)=\sum_{m=-\infty}^{\infty} H_{m}(\rho) e^{i m \phi}
\end{aligned}
$$

Substituting these expansions in 2.19 and 2.20 and using standard Fourier series methods,

$$
\begin{aligned}
& \frac{d^{2} E_{m}(\rho)}{d \rho^{2}}+\left(\frac{1}{\rho}-\frac{1}{\mu(\rho)} \frac{d \mu(\rho)}{d \rho}\right) \frac{d E_{m}(\rho)}{d \rho}+\left(k^{2}(\rho)-\frac{m^{2}}{\rho^{2}}\right) E_{m}(\rho)=0 \\
& \frac{d^{2} H_{m}(\rho)}{d \rho^{2}}+\left(\frac{1}{\rho}-\frac{1}{\varepsilon(\rho)} \frac{d \varepsilon(\rho)}{d \rho}\right) \frac{d H_{m}(\rho)}{d \rho}+\left(k^{2}(\rho)-\frac{m^{2}}{\rho^{2}}\right) H_{m}(\rho)=0
\end{aligned}
$$

For the remainder of this subsection only the E polarization case will be discussed, since it is clear that the further development of the $H$ polarization case will follow a completely analogous line.

$$
\text { Now for } \rho>\rho_{c} \text {, }
$$




$$
\frac{d \mu(\rho)}{d \rho}=0
$$

and with an obvious notation

$$
k^{2}(p)=k_{c}^{2}
$$

2.23 anà 2.24 both reduce to Bessel's equation.

In this constant $\mathrm{k}$ region it is possible to write

$$
E_{m}(\rho)=E_{m}^{\text {inc }}(\rho)+E_{m}^{\text {scat }}(\rho)
$$

where, since

$$
\begin{aligned}
E^{i n c}(\rho, \phi) & =E_{0} e^{i k \rho \cos \phi} \\
& =E_{0} \sum_{m=-\infty}^{\infty} i^{m} J_{m}\left(k_{c} \rho\right) e^{i m \phi}
\end{aligned}
$$

it is clear that

$$
E_{m}^{i n c}(\rho)=E_{o} i^{m} J_{m}\left(k_{c} \rho\right)
$$

and where, since $\mathrm{E}_{\mathrm{m}}^{\mathrm{Scat}}(\rho)$ must represent the $\mathrm{m}$ component of an outgoing wave for $\rho \rightarrow \infty$, it is also clear that

$$
E_{m}^{\text {scat }}(\rho)=E_{0} i^{m_{R}}{ }_{m} H_{m}\left(k_{c} \rho\right)
$$

in which $R_{m}$ is some, as yet undetermined, constant $\left(R_{m}=R_{-m}\right)$ which may be termed the model reflection coefficient. This coefficient will be required frequently in the following. It should be noted that in the above equation, and for the rest of this report, a nugatory superscript on the Hankel function symbol will be omitted; that is to say,

$$
H_{m}(x) \equiv H_{m}^{(I)}(x)
$$


If a solution to equation 2.23 which remained finite at $\rho=0$ were known for $\rho<\rho_{c}$, the solution 2.25 must be the continuation of this solution into the region $\rho>\rho_{c}$. Let the correct solution for $\rho<\rho_{c}$ be denoted by $F_{m}(\rho)$ then

$$
\begin{aligned}
& F_{m}\left(\rho_{c}\right)=E_{o} i^{m}\left[J_{m}\left(k_{c} \rho_{c}\right)+R_{m} H_{m}\left(k_{c} \rho_{c}\right)\right] \\
& \left.\frac{d F_{m}(\rho)}{d \rho}\right|_{\rho=\rho_{c}}=E_{o} i^{m_{k}}\left[J_{m}^{\prime}\left(k_{c} \rho_{c}\right)+R_{m} H_{m}^{\prime}\left(k_{c} \rho_{c}\right)\right]
\end{aligned}
$$

Rearranging,

$$
R_{m}=-\left\{\frac{F_{m}\left(\rho_{c}\right) J_{m}^{\prime}\left(k_{c} \rho_{c}\right)-\left.\frac{1}{k_{c}} \frac{d F_{m}(\rho)}{d \rho}\right|_{\rho=\rho_{c}} \cdot J_{m}\left(k_{c} \rho_{c}\right)}{F_{m}\left(\rho_{c}\right) H_{m}^{\prime}\left(k_{c} \rho_{c}\right)-\left.\frac{I}{k_{c}} \frac{d F_{m}(\rho)}{d \rho}\right|_{\rho=\rho_{c}} \cdot H_{m}\left(k_{c} \rho_{c}\right)}\right\}
$$

which, it should be noticed, is homogeneous in $F_{m}$.

Having determined the $R_{m}$, we may write the scattered field in the explicit form, for $\rho>\rho_{c}$ :

$$
E^{\text {scat }}(\rho, \varnothing)=E_{0} \sum_{m=-\infty}^{\infty} i^{m_{R_{m}} H_{m}\left(k_{c} \rho\right) e^{i m \varnothing}}
$$

Using the asymptotic forms of the Hankel functions, the scattered field for large $\rho$ assumes the form: 


$$
\begin{aligned}
& E^{\text {scat }}(\rho, \phi)=E_{0} \sqrt{\frac{2}{\pi k c_{c} i}} \cdot e^{i k c^{\rho}} \sum_{m=-\infty}^{\infty} R_{m} e^{i m \phi} \\
& E^{\text {scat }}(\rho, \phi)=E_{0} \sqrt{\frac{2}{\pi k} k_{c}} e^{i\left(k_{c} \rho-\pi / 4\right)} \sum_{m=0}^{\infty} \varepsilon_{m} R_{m} \cos m \phi
\end{aligned}
$$

where $\varepsilon_{0}=1 ; \quad \varepsilon_{m}=2, \quad m \geqq 1$. Defining a "scattering width"

$$
\sigma_{2} \equiv \frac{\frac{1}{2} \operatorname{Re} \int E_{z}^{\text {scat }} H_{\phi}^{\text {scat }} \rho d \emptyset}{\frac{1}{2} \operatorname{Re} E_{z}^{i n c} H_{y}^{* i n c}}
$$

and using 2.26

$$
\sigma_{2}=\frac{2}{k_{c}}\left\{\left|R_{o}\right|^{2}+4 \sum_{I}^{\infty}\left|R_{m}\right|^{2}\right\}
$$

As an example of the above formalism one may take the "twodimensional Luneberg lens" configuration studied by Jasik (I). For that case

$$
\begin{aligned}
& \mu(\rho)=\mu\left(\rho_{c}\right) \\
& \varepsilon(\rho)=\sqrt{2-\frac{\rho^{2}}{\rho_{c}^{2}}} \varepsilon_{c}
\end{aligned}
$$

allowing us to write the equation for $F_{m}$ in the form:

$$
\frac{d^{2} F_{m}(\rho)}{d \rho^{2}}+\frac{1}{\rho} \frac{d F_{m}(\rho)}{d \rho}+\left(2 k_{0}^{2}-\frac{k_{o}^{2} \rho^{2}}{\rho_{c}^{2}}-\frac{m^{2}}{\rho^{2}}\right) F_{m}(\rho)=0
$$

of which the solution is 


$$
F_{m}(\rho)=A_{m} \rho^{m} e^{\frac{k \rho^{2}}{2 \rho} c} F_{I}\left(-\frac{k a}{2}+\frac{m+1}{2}, m+1, \frac{k \rho^{2}}{\rho_{c}}\right)
$$

where $A_{m}$ is a constant.

For later comparison we note that Jasik considers the far field of a line source at the boundary and so sums:

$$
E^{\operatorname{scat}}(\rho, \varnothing) \sqrt{\frac{\pi k c^{\rho}}{2}} e^{-i\left(k c^{\rho}-\frac{\pi}{4}\right)}=\frac{I \omega \mu}{4} \sum_{m=0}^{\infty} \varepsilon_{m} R_{m} \frac{H_{m}\left(k_{c} \rho c\right)}{(i)} \cos m \varnothing
$$

where $I$ is the current in the line source.

Of course, equation 2.23 may be integrated in terms of wellstudied functions for other particular cases of $\rho$ variation of the medium parameters. For example if

$$
\begin{aligned}
& \mu(\rho)=\mu\left(\rho_{c}\right) \\
& \varepsilon(\rho)=\varepsilon\left(\rho_{c}\right) \rho^{2} / a^{2}
\end{aligned}
$$

then

$$
F_{m}^{\prime \prime}+\frac{F_{m}^{\prime}}{\rho}+\left(k_{c}^{2} \frac{\rho^{2}}{\rho_{c}^{2}}-\frac{m^{2}}{\rho^{2}}\right) F_{m}=0
$$

of which the solution is

$$
F_{m}=A_{m} J_{m / 2}\left(\frac{k_{c} \rho^{2}}{2 \rho_{c}}\right)
$$

and again $A_{m}$ is an arbitrary constant.

In general, however, equation 2.23 must be integrated numerically. The point of Section 3 of this report is to reduce the numerical work which would result from a straightforward application of the formalism of this section. 
A formalism similar to that given above carries through for the spherically stratified case. The situation in that case is complicated by the fact that when an incident plane wave-excites waves in the sphere, these waves are analogous to a combination of the two polarizations treated above. More about this will be said in Section 3 . Our purpose here has merely been to explore how far the separation of variables method could take us in the simplest case of interest.

B. Thin Shell Approximation

We turn now to another standard method of solving stratified inhomogeneity problems.

The basic assumption in the "thin-shell" approximation is that the stratified inhomogeneity may be conceived of as being made up of a large number of shells of uniform thickness, although the thickness may vary from shell to shell. The parameters of the medium are assumed not to vary within each individual shell.

We continue, as in the latter part of Section 2A, to speak only of the E polarization case for the cylinder. The situation is diagrammed in Figure 2, for which the only component of electric field is $E_{Z}(\rho, \phi)$, and in which the $(N+1)^{\text {st }}$ medium is identified with the exterior region.

As in the previous section, an incident wave is assumed; for instance the plane wave

$$
\mathrm{E}_{\mathrm{z}}^{\mathrm{inc}}(\rho, \phi)=\mathrm{E}_{0} \sum_{0}^{\infty} \varepsilon_{\mathrm{m}} i^{\mathrm{m} J_{\mathrm{m}}}\left(\mathrm{k}_{\mathrm{c}} \rho\right) \cos \mathrm{m} \phi
$$

In each of the shells the equation which $E_{z}(\rho, \varnothing)$ satisfies is simply 


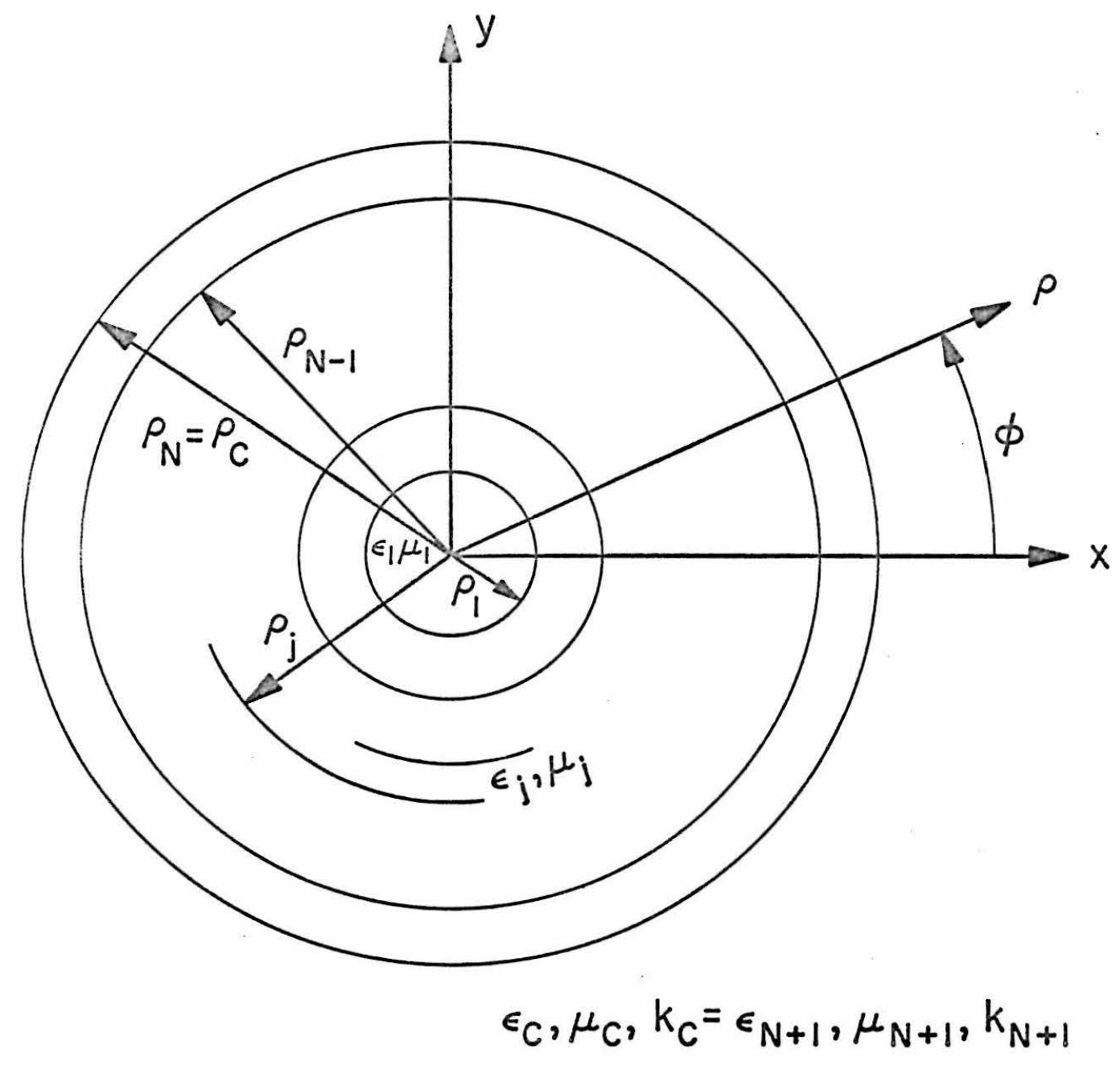

Fig. 2. Cross-section of a cylindrically symmetric inhomogeneity as treated by the thin shell approximation 
the Helmholtz equation with the $k$ appropriate to that shell.

$$
\left(\nabla^{2}+k_{j}^{2}\right) E_{z}^{j}(\rho, \varnothing)=0
$$

Taking into account the angular variation of the incident wave we may write the solution of 2.28 in the form

$$
\begin{array}{r}
E_{z}^{j}(\rho, \varnothing)=E_{0} \sum_{m=0}^{\infty}\left[A_{j}^{m} J_{m}\left(k_{j} \rho\right)+R_{j} H_{m}\left(k_{z} \rho\right)\right] \cos m \varnothing, \\
j=1, \cdots N+1,
\end{array}
$$

where, to satisfy the condition of finiteness at the origin we must put

$$
R_{1}^{m}=0
$$

and to conform with our partial knowledge of the field in the exterior region we must have

$$
A_{N+1}^{m}=\varepsilon_{m} i^{m}
$$

Now using the orthogonal properties of the trigonometric functions and the continuity of the tangential components of the $\mathrm{E}$ and $\mathrm{H}$ fields at each boundary between shells, we may write

$$
\begin{aligned}
& A_{j}^{m} J_{m}\left(k_{j} \rho_{j}\right)+R_{j}^{m} H_{m}\left(k_{j} \rho_{j}\right)=A_{j+I}^{m} J_{m}\left(k_{j+1} \rho_{j}\right)+R_{j+1}^{m} H_{m}\left(k_{j+1} \rho_{j}\right) \\
& A_{j}^{m} J_{m}^{\prime}\left(k_{j} \rho_{j}\right)+R_{j}^{m} H_{m}^{\prime}\left(k_{j} \rho_{j}\right)=\frac{\mu_{j} k_{j+I}}{k_{j}^{\mu}{ }_{j+1}}\left\{A_{j+I}^{m} J_{m}^{\prime}\left(k_{j+1} \rho_{j}\right)+R_{j+1}^{m} H_{m}^{\prime}\left(k_{j+1} \rho_{j}\right)\right\} \\
& j=1, \cdots N
\end{aligned}
$$


For the $\mathrm{H}$ polarization case equations exactly analogous to these are obtained in which $\mu_{j}$ is replaced by $\varepsilon_{j}$ in the factors multiplying the right hand side of equation 2.30 .

Equations 2.29 and 2.30 are $2 N$ equations in the $2 N$ unknowns:

$$
\begin{aligned}
& \mathrm{A}_{I}^{\mathrm{m}} \\
& \mathrm{A}_{j}^{\mathrm{m}}, \mathrm{R}_{j}^{\mathrm{m}} \quad(j=2,3, \cdots N) \\
& \mathrm{R}_{\mathrm{N}+1}^{\mathrm{m}} .
\end{aligned}
$$

A fairly simple method of solution of equations 2.29 and 2.30 is the following.

First solve these equations for $R_{j+1}, A_{j+1}$ in terms of $R_{j}$ and $A_{j}$. Then use the Wronskian relations for Bessel functions to reduce these equations to the form

$$
A_{j+1}^{m} A_{j+1}^{m}=\left(\begin{array}{cc}
{ }_{A A}^{m, j} & { }_{A R}^{m, j} \\
M_{R A}^{m, j} & M_{R R}^{m, j}
\end{array}\right) \quad A_{j}^{m}
$$

where

$$
\begin{aligned}
& \mathrm{N}_{\mathrm{AA}}^{j}=\frac{\pi \rho_{j}}{2 i \mu_{j}}\left\{\mu_{j} k_{j+I} J_{m}\left(k_{j} \rho_{j}\right) H_{m}^{\prime}\left(k_{j+I}, \rho_{j}\right)-k_{j} \mu_{j+1} J_{m}^{\prime}\left(k_{j} \rho_{j}\right) H_{m}^{\prime}\left(k_{j+I} \rho_{j}\right)\right\} \\
& M_{A R}^{m, j}=\frac{\pi \rho_{j}}{2 i \mu_{j}}\left\{\mu_{j} k_{j+1} H_{m}\left(k_{j} \rho_{j}\right) H_{m}^{\prime}\left(k_{m+1} \rho_{j}\right)-k_{j} \mu_{j+1} H_{m}^{\prime}\left(k_{j} \rho_{j}\right) H_{m}\left(k_{j+1} \rho_{j}\right)\right\} \\
& M_{R A}^{m, j}=-\frac{\pi \rho_{j}}{2 i \mu_{j}}\left\{\mu_{j} k_{j+I} J_{m}\left(k_{j} \rho_{j}\right) J_{m}^{\prime}\left(k_{j+I} \rho_{j}\right)-k_{j} \mu_{j+I} J_{m}^{\prime} \cdot\left(k_{j} \rho_{j}\right) J_{m}\left(k_{j+I} \rho_{j}\right)\right\}
\end{aligned}
$$




$$
M_{R R}^{m, j}=-\frac{\pi \rho_{j}}{2 i \mu_{j}}\left\{\mu_{j} k_{j+1} H_{m}\left(k_{j} \rho_{j}\right) J_{m}^{\prime}\left(k_{m+1} \rho_{j}\right)-k_{j} \mu_{j+1} H_{m}^{\prime}\left(k_{j} \rho_{j}\right) J_{m}\left(k_{j+1} \rho_{j}\right)\right\}
$$

Now equations 2.31 may be used to calculate consecutively the coefficients in terms of $A_{l}^{m}$ and $R_{l}^{m}$. Since

$$
R_{1}^{m}=0
$$

we temporarily assume

$$
A_{1}^{m}=1
$$

and calculate

$$
\left(\begin{array}{c}
A_{N+1}^{m} \\
R_{N+1}^{m}
\end{array}\right)=\left(M^{m, N} \cdot M^{m, N-1} \cdots \cdot M^{m, 2} \cdot M^{m, I}\right)\left(\begin{array}{l}
1 \\
0
\end{array}\right)
$$

Since this equation is linear in $A_{N+1}^{m}, R_{N+1}^{m}$ the modal reflection coefficient for substitution in such equations as 2.26 may be calculated simply by the formula

$$
R_{m}=\frac{R_{N+1}^{m}}{A_{N+1}^{m}} \cdot(i)^{n}
$$

One difficulty with this method is that of calculating the matrix elements of equation 2.31 with any accuracy. This is because of the subtraction of functions which are almost equal when $\left|\rho_{j+1}-\rho_{j}\right|$ becomes small.

A further difficulty is that of establishing the correctness of the limiting solution which is obtained by this method as the number of shells is increased and $\max \left|\rho_{j+1}-\rho_{j}\right| \rightarrow 0$. The entire reflection process in the above approximation is due to the discontinuities 
of $k$ while in the actual case $k$ may be continuous.

We will see in the third section how these difficulties are overcome in the reformulation of the problem which is made there.

The method of Section $2 B$ carries through completely for the spherically stratified medium. As in section $2 \mathrm{~A}$, our purpose here has been merely to exhibit a method of computation in its most transparent form.

C. Invariant Imbedding in Plane Stratified Media

This subsection is a short exposition of the method of invariant imbeding, as it applies to wave problems, using as an example the simplest interesting case for which the method is useful; that of the plane stratified medium.

The purpose of this, for by now the applications of the method in the plane case are well known, is to exhibit the simplicity of the basic ideas of the method, unadulterated by the cumbersome formulas which arise in the cylindrical and spherical stratified media. The concept of "invariant imbedding", which is not a very enlightening title, is a generalization of a principle originally enunciated by Ambarzumian (2) in connection with the diffuse reflection of light by a foggy medium. The first form of Ambarzumian's principle was that the intensity distribution of light emerging from a semiinfinite foggy medium, or of incident light reflected by this medium, is unchanged by the addition of a small extra layer of fog at the surface. This is clear in Ambarzumian's case, since using the concept of optical length the stratified medium in the optical intensity 
approximation may be reduced effectively to a homogeneous medium. For a homogeneous medium the small additional layer may be thought of, alternatively, as a coordinate transformation which cannot change the emerging intensity

Since Ambarzumian's work, principles of invariance have been formulated which allow a similar treatment of reflection of light by a slab of foggy medium of finite thickness. These extensions have been largely due to Chandrasekhar (3).

The name "invariant imbedding" for this type of problem seems to have been popularized by Bellman $(4,5)$ who conceived of the method as that of associating the problem of which we want the solution to a large class of similar problems. If we restrict our desire to calculating some particular parameter (or functional, in slightly more mathematical language) of the solutions, we may be able to prescribe a method of calculating this parameter for a large number of cases without obtaining the complete solution in each case.

For example, Ambarzumian's method in the language of invariant imbedding would be as follows. We wish to obtain the reflection coefficient of a particular semi-infinite foggy medium. To do this we imbed our particular foggy medium in a large class of similar media distinguished by the particular plane in space at which they start. We now obtain an equation for the rate of change of reflected light as we change some parameter labeling the medium giving that amount of reflection. This is the main point of the method, and it turns out that it is often quite straightforward, using physical arguments to derive the equation. In the foggy medium case, one--but by no means the only--way 
of deriving it is by invoking Ambarzumian's invariance principle. Once this rate of change equation has been formulated with an appropriate initial condition, it may be used to calculate the parameter desired in a large number of cases; for example, the reflection coefficient of slabs of a foggy medium of various widths. Since in many situations of technical importance it is just this variation of some parameter as we change the physical set-up which is interesting, a great deal of computation has been avoided completely by concentrating solely on the parameter of interest.

Because of the usefulness of invariance principles in formulating the rate-of-change equation, the principles of invariance and those of invariant imbedding have sometimes been confused. It is hoped that the above will help alleviate this confusion of language. In summary, invariant imbedding is an approach to physical problems almost too general to be useful, while principles of invariance are important enough tools to make the invariant imbeding approach of practical use. The first application of principles of invariance to wave problems appears to have been made by Papas (6) when he calculated the reflection coefficient of a plane inhomogeneous dielectric slab when an electromagnetic wave is incident. We will now give what, essentially, is a summary of Papas' approach, with the problem slightly generalized to include non-uniform permeability.

Subsequently we will give other derivations of the rate of change equation, demonstrating the logical distinction between principles of invariance and invariant imbedding. 
We consider the situation of Figure 3 in which a plane electromagnetic wave is normally incident, from a region in which the constitutive parameters are uniform onto a semi-infinite region in which these parameters vary only in one direction and become again uniform at some depth, say $z=b$. That is, we have

$$
\begin{array}{lll}
\varepsilon=\varepsilon_{1} & \mu=\mu_{1} & z<0 \\
\varepsilon=\varepsilon_{2} & \mu=\mu_{2} & z>b \\
\varepsilon=\varepsilon_{n}(z) & \mu=\mu_{n}(z) & b>z>0
\end{array}
$$

where $\varepsilon$ and $\mu$ are not necessarily continuous at $z=0$ and $z=b$. To talk about this problem in the language of invariant imbedding we consider it as being one of a class of problems, each of which has the same $\varepsilon$ and $\mu$ as a function of $z$ for $z>a$, where $b>a>0$ and where $\varepsilon_{a}(z)=\varepsilon(a)$ and $\mu_{a}(z)=\mu(a)$ for $z<a$.

Now a plane electromagnetic wave with components $E_{x}$ and $H_{y}$ is assumed to be incident from the left in each problem distinguished by the label a. That is to say, we have

$$
\begin{aligned}
& E_{x}^{a}=e^{i k a^{z}}+R_{a} e^{-i k a^{z}} \\
& H_{y}^{a}=\frac{1}{z_{a}}\left(e^{i k a^{z}}-R_{a} e^{-i k a^{z}}\right)
\end{aligned}
$$

where

$$
\begin{aligned}
& \mathrm{z}_{\mathrm{a}}=\sqrt{\mu(\mathrm{a}) / \varepsilon(\mathrm{a})} \\
& \mathrm{k}_{\mathrm{a}}=\omega \sqrt{\mu(\mathrm{a}) \varepsilon(\mathrm{a})}
\end{aligned}
$$




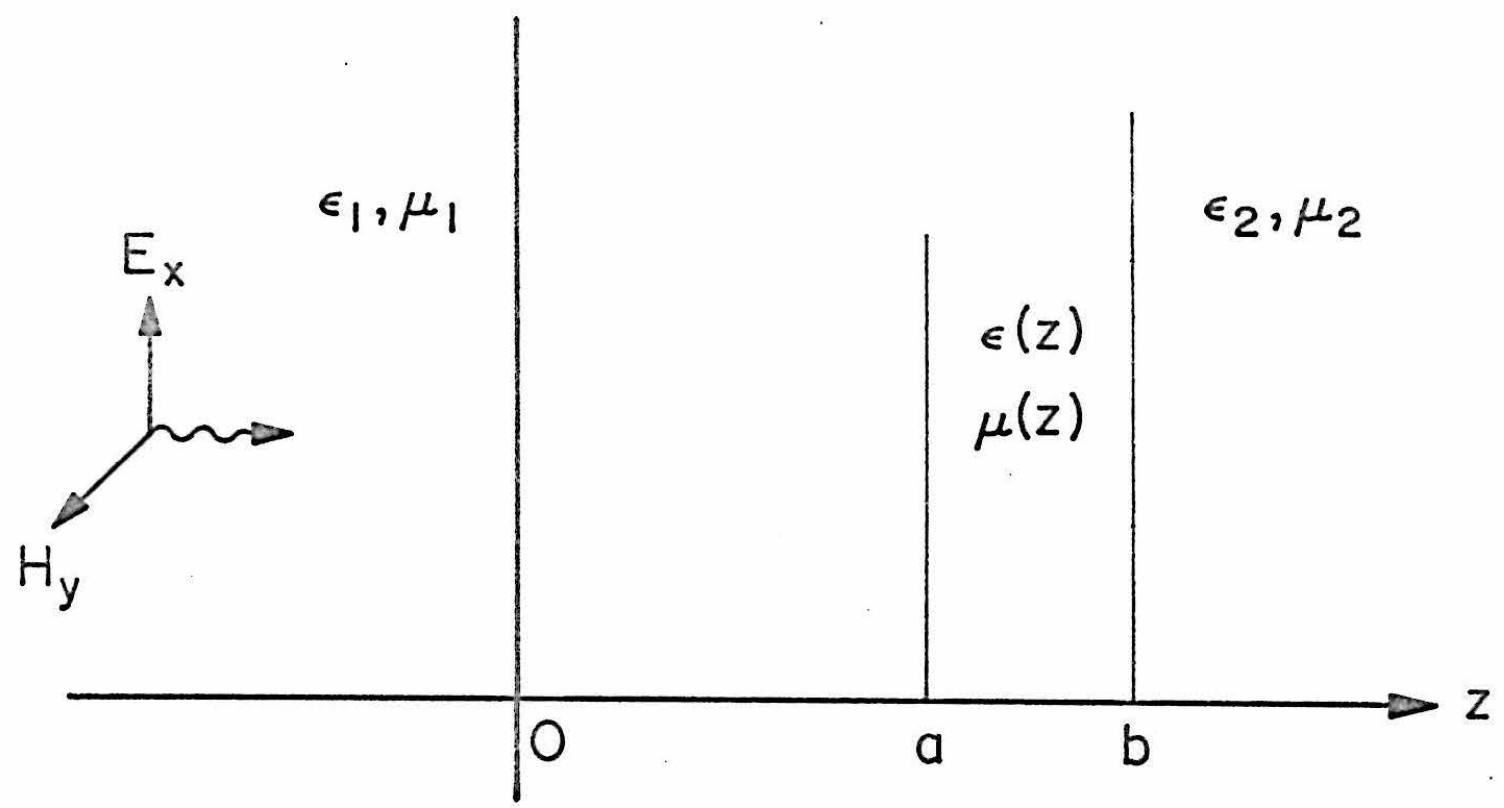

Fig. 3. A one-dimensional inhomogeneity problem treated by the method of invariant imbedding

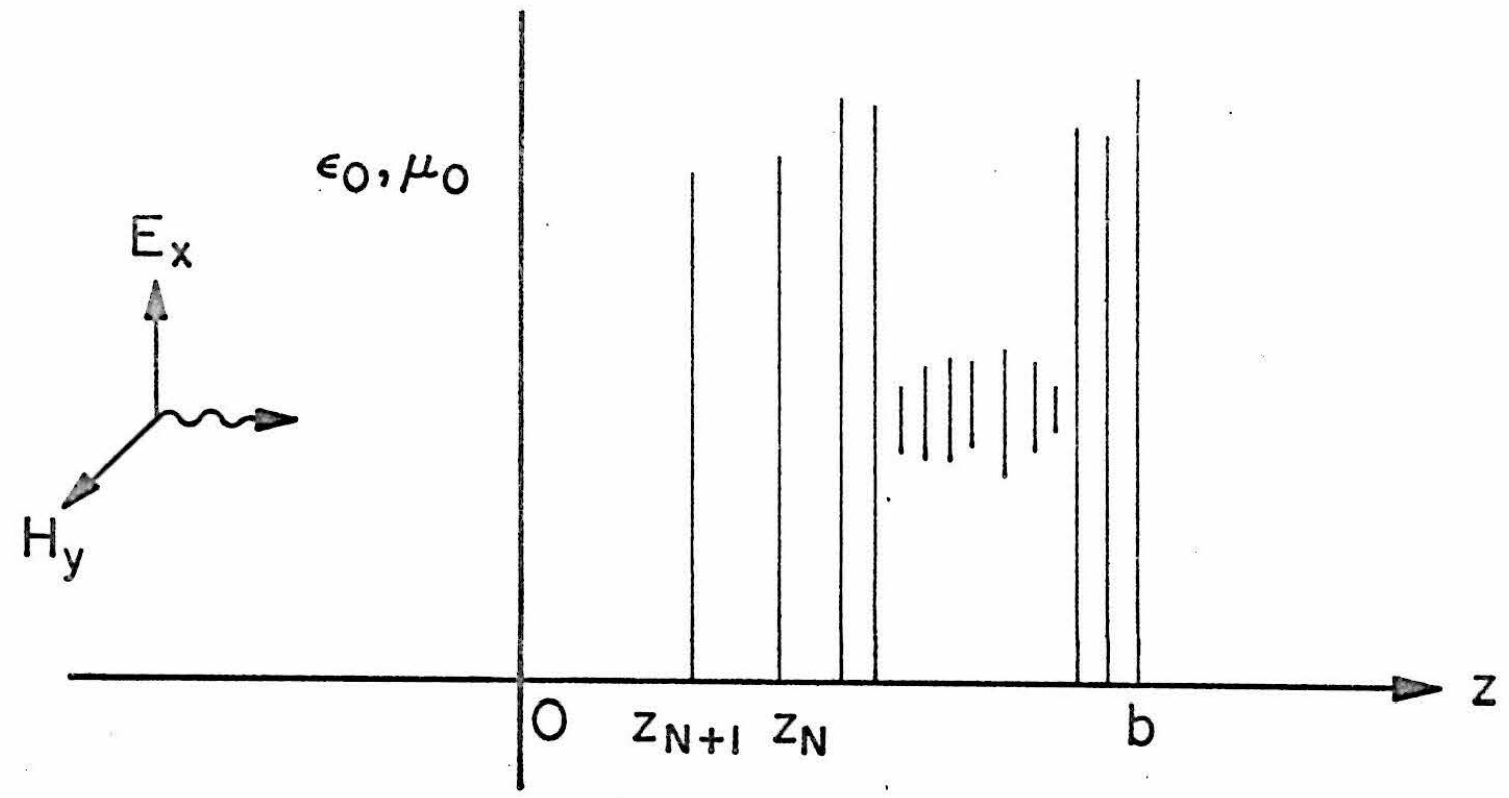

Fig. 4. A one-dimensional inhomo-geneity problem treated by the thin slab approximation 
The primary interest in this case is in the reflection coefficient $R_{a}$. In particular we would like to calculate $R_{0}$ but if a rate of change equation can be derived for $R_{a}$ we will obtain, with no additional effort, the reflection coefficients of a large class of inhomogeneous slabs which may be of interest.

To derive this rate of change equation we use the notion, easily acceptable on physical grounds, that to go from the situation for which $a=a_{1}+\Delta$ to that for which $a=a_{1}$ we merely change the constitutive parameters for $z<a_{1}$ from those associated with $a_{1}+\Delta$ in the final configuration to those associated with $a_{1}$. If the increment $\Delta$ is small the effect of the above change on the reflection coefficient may be taken into account by considering only the first few reflections which one calculates when applying the "method of multiple reflections" to this problem.

In particular

$$
\mathrm{R}_{\mathrm{a}_{1}} \simeq \mathrm{R}_{1}+\mathrm{R}_{2}+\mathrm{R}_{3} \cdots
$$

where $R_{1}$ is the reflection which occurs due only to the interface at $z=a_{1}, R_{2}$ accounts for that field which is transmitted at $z=a_{1}$ but reflected (with reflection coefficient $\mathrm{R}_{\mathrm{a}_{1}+\Delta^{\text {) }}}$ at $a_{1}+\Delta$ and then is retransmitted through the $z=a_{1}$ interface. Similarly $R_{3}$ accounts for three internal reflections in the incremental slab.

Now we define $r(x, y)$ as the reflection coefficient when a wave is incident from a medium with constitutive parameters corresponding to position $z=x$ in our final problem, on a medium with these 
parameters corresponding to the position $\mathrm{z}=\mathrm{y}$ in the final problem. Using the definition

$$
\mathrm{z}(\mathrm{z})=\sqrt{\mu(\mathrm{z}) / \varepsilon(\mathrm{z})}
$$

one may show by solving the elementary boundary value problem involved that

$$
\begin{aligned}
& r(x, y)=-\left(\frac{Z(x)-Z(y)}{Z(x)+Z(y)}\right) \\
& r(y, x)=-r(x, y)
\end{aligned}
$$

With a definition of transmission coefficient $t(x, y)$ similar to that for $r(x, y)$ it is also easy to show that

$$
\begin{aligned}
& t(x, y)=1+r(x, y) \\
& t(y, x)=1+r(y, x)
\end{aligned}
$$

Now, bearing in mind the paragraph immediately following equation 2.32, it is clear that

$$
R_{1}=r\left(a_{1}-0, a_{1}+\Delta-0\right)
$$

by which we mean that

$$
R_{1}=\lim _{\varepsilon \rightarrow 0} r\left(a_{1}-\varepsilon, a_{1}+\Delta-\varepsilon\right)
$$

This limiting process takes into account the fact that $\varepsilon(\mathrm{z})$ and $\mu(z)$ may be discontinuous from the left. Also, it is clear from the above definitions that 
$R_{2}=t\left(a_{1}-0, a_{1}+\Delta-0\right) e^{i k a_{1}+\Delta-0^{\Delta}} R_{a_{1}+\Delta} e^{i k a_{1}+\Delta-0 \Delta} t\left(a_{1}+\Delta-0, a_{1}-0\right)$

$R_{n+1}=R_{n} r\left(a_{1}+\Delta-0, a_{1}-0\right) e^{i k a_{1}+\Delta-0 \Delta} R_{a_{1}+\Delta} e^{i k a_{1}+\Delta-0 \Delta}, n \geqslant 2$

Because of the recursion relation 2.35 , it is possible to sum the whole series implicit in equation 2.33 in the form:

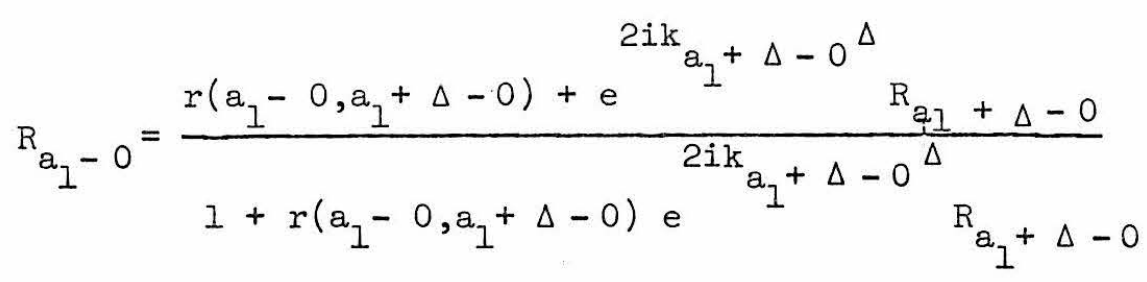

This equation is of interest mainly when $\Delta \rightarrow 0$ so that we have

$$
R_{a_{1}-0}=\frac{r\left(a_{1}-0, a_{1}+0\right)+R_{a_{1}}+0}{1+r\left(a_{1}-0, a_{1}+0\right) R_{a_{1}}+0}
$$

Returning to our expressions for the first few reflections and expanding these to first order in $\Delta$, since $\Delta$ is a small quantity, we obtain

$$
\begin{aligned}
& R_{1} \rightarrow+z^{\prime}\left(a_{1}\right) \frac{\Delta}{2 Z\left(a_{1}\right)} \\
& R_{2} \rightarrow R_{a_{1}}+R_{a_{1}^{\prime}} \Delta+2 i k\left(a_{1}\right) R_{a_{1}} \\
& R_{3} \rightarrow R_{a_{1}}^{2} Z^{\prime}\left(a_{1}\right) \frac{\Delta}{2 Z} \\
& R_{a_{1}}^{\prime}=d R_{a} /\left.d a\right|_{a=a_{1}}
\end{aligned}
$$


These results can be combined with equation 2.33 to give

$$
R_{a}^{\prime}=-\frac{Z^{\prime}(a)}{2 Z(a)}\left(I-R_{a}^{2}\right)-2 i k(a) R_{a}
$$

Equation 2.38 is a Riccati equation which may be integrated with ease, numerically. The integration starts with $a=b$ and the initial condition is

$$
R_{b}=r(b-0, b+0)
$$

If there are a finite number of discontinuities in the medium, equation 2.37 may be employed at each one as the integration proceeds backwards in. a . Thus any discontinuity at $a=0$ is automatically included in the integration in the same manner as any jump in parameters within the slab.

Non-normal incidence may be treated in a manner exactly analogous to that given above but it would seem more useful for an understanding of the method to demonstrate other ways of deriving the rate of change equation 2.38 .

One way of proceeding would be to consider the situation diagrammed in Figure 4. In this figure the inhomogeneity is considered to consist of a large number of thin homogeneous slabs. By employing the procedure described in Section $2 B$ and by applying some transformations to the equation analogous to 2.31 which results, one may derive the equation which we now obtain by a shorter route. Referring to Figure 4 we now wish to derive the reflection coefficient at plane $z_{n+l}$ (assuming the medium to the left of this 
plane is homogeneous) from the reflection coefficient at plane $z_{n}$, which is assumed to be known.

This is a straightforward boundary value problem and we obtain, of course, in agreement with equation 2.36

$R_{n+1}+R_{n+1} R_{n}\left(\frac{z_{n}-z_{0}}{z_{n}+z_{0}}\right) e^{2 i k_{n}\left(z_{n}-z_{n+1}\right)}=\frac{z_{n}-z_{0}}{z_{n}+z_{0}}+e^{2 i k_{n}\left(z_{n}-z_{n+1}\right)} R_{n}$

where $k_{n}, z_{n}$ are the propagation constant and impedance associated with the slab between $z_{n}$ and $z_{n+l}$ while $z_{0}$ is the impedance of the homogeneous medium on the left.

Now, in approximating a medium whose constitutive parameters vary continuously, we may take a large number of slabs and use the approximations:

$$
\begin{aligned}
& \frac{z_{n}-z_{0}}{z_{n}+z_{0}}=\frac{Z^{\prime}\left(z_{n}\right)}{2 Z\left(z_{n}\right)}\left(z_{n}-z_{n+1}\right) \\
& R_{n+1}=R+\left(z_{n}-z_{n+1}\right) R^{\prime}\left(z_{n}\right) \\
& R_{n}=R
\end{aligned}
$$

Substituting these approximations in the preceding equation and then letting $z_{n} \rightarrow z_{n+1}$ we again obtain equation 2.38 .

Of course the above method is quite similar to the previous one. It merely avoids the use of multiple reflections in order to demonstrate that the method is not inherently connected to the method of invariant imbedding. Both these methods, however, are well connected with 
physical ways of reasoning, and that is a most important point about the method of imbedding--that physical reasoning can often derive easily the basic rate-of-change equation involved.

As a contrast with this we will now demonstrate a purely formal way of deriving equation 2.38. The following cannot be recommended as a method in other problems, since so little of an intuitive nature is involved, but it does show that everything in the invariant imbedding method is built into the basic differential equations of the problem. All that is required is sufficient ingenuity to make the "right" transformations.

First we note from equation 2.14 that for the present problem, using $\mathrm{E}_{\mathrm{x}}$ as our dependent variable, the differential equation which must be solved is:

$$
\frac{d^{2}}{d z^{2}} E_{x}(z)+k^{2}(z) E_{x}(z)-\frac{1}{\mu(z)} \frac{d \mu(z)}{d z} \frac{d E_{x}(z)}{d z}=0
$$

We also note, as may easily be shown from the definitions of $k(z)$ and $z(z)$, that

$$
\frac{1}{\mu(z)} \frac{d \mu(z)}{d z}=\frac{1}{k(z)} \frac{d k(z)}{d z}+\frac{1}{z(z)} \frac{d z(z)}{d z}
$$

Now let us consiaer the function

$$
F(z)=\frac{i k(z) E_{x}(z)-\frac{d}{d z} E_{x}(z)}{i k(z) E_{x}(z)+\frac{d}{d z} E_{x}(z)}
$$

By direct differentiation 


$$
\frac{d F(z)}{d z}=-2 i k \frac{\frac{1}{E_{x}} \frac{d^{2} E_{x}}{d z^{2}}-\left(\frac{1}{E_{x}} \frac{d E_{x}}{d z}\right)^{2}-\frac{1}{k} \frac{d k}{d z} \frac{1}{E_{x}} \frac{d E_{x}}{d z}}{\left(i k-\frac{1}{E_{x}} \frac{d E_{x}}{d z}\right)^{2}}
$$

Substituting from 2.39 for the second derivative term, and employing 2.40, one can show, after some algebraic manipulation that

$$
\frac{d F(z)}{d z}=-\frac{Z^{\prime}}{2 Z}\left(I-F^{2}(z)\right)-2 i k F(z)
$$

Equation 2.42 is of the same form as equation 2.38 and since, from an examination of equation 2.41, the functions $F$ and $R$ satisfy the same boundary condition at $\mathrm{z}=\mathrm{b}$, we may identify $\mathrm{F}(\mathrm{z})$ with $R_{z}$ and state that $F(0)=R_{0}$. Also, by further work with equation 2.41, one can obtain an equation analogous to equation 2.37 which is applicable when there are discontinuities in the medium.

It should be noted in passing that if it is desirable to calculate transmission coefficients as well as reflection coefficients, there are two paths one can follow. One method is to calculate the reflection coefficient for a wave incident from the right on our layered medium (this will be denoted by $\overleftarrow{R}_{a}$ ) as a function of position, and then to employ the equations

$$
\begin{aligned}
& \frac{d T(z)}{d z}=\left(1+\frac{t}{R}\right) \frac{Z^{\prime}(z)}{2 Z(z)} T(z)+i k(z) T(z) \\
& T(0-)=1 ;
\end{aligned}
$$

then the coefficient we desire is $T(a)$. 
With the second method we integrate the equation for $T$ in the same direction as that for $R$ so that both integrations may be carried out simultaneously on a computing machine. The equations to be used in this case are

$$
\begin{aligned}
& \frac{\mathrm{dT}(\mathrm{z})}{\mathrm{dz}}=+\frac{\mathrm{Z}^{\prime}(\mathrm{z})}{2 \mathrm{Z}(\mathrm{z})}\left(1-\mathrm{R}_{\mathrm{z}}\right)+i k(z) T(z) \\
& \mathrm{T}(\mathrm{a}+)=1
\end{aligned}
$$

and the number we want is $\mathrm{T}\left(\mathrm{O}_{-}\right)$.

Both the above equations may be derived in a straightforward manner using the multiple reflection method. Obviously the second method is more appropriate if waves are incident only from one direction.

The method of treating discontinuities is also a straightforward result of the multiple reflection method and may be stated in the forms

$$
\begin{aligned}
& T\left(z_{n}+0\right)=\frac{2 Z\left(z_{n}+0\right) T\left(z_{n}-0\right)}{\left[Z\left(z_{n}+0\right)+Z\left(z_{n}-0\right)\right]-\left[Z\left(z_{n}+0\right)-Z\left(z_{n}-0\right)\right] \hat{R}_{z_{n}}^{\leftarrow}-0} \\
& T\left(z_{n}-0\right)=\frac{2 Z\left(z_{n}+0\right) T\left(z_{n}+0\right)}{\left[Z\left(z_{n}+0\right)+Z\left(z_{n}-0\right)\right]+\left[Z\left(z_{n}+0\right)-Z\left(z_{n}-0\right)\right] R_{z_{n}}+0}
\end{aligned}
$$

for the first and second methods, respectively, where $z_{n}$ is the position of a discontinuity in the medium.

We also note here, as a matter of interest, that via some algebraic manipulation on equation 2.39 it may be shown that if the phases of the transmission coefficients in each direction are properly 
chosen, a simple relation exists between them. More explicitly, we write

$$
E_{x}(z)=T e^{i k_{2} z}, \quad z>b
$$

for a wave incident from the left of the form $e^{i k_{1} z}$, and further we write

$$
E_{x}(z)=\stackrel{+}{T} e^{-i k_{l} z}, \quad z<0
$$

for a wave incident from the right of the form $e^{-i k_{2} z}$.

Now if $\phi_{1}$ and $\phi_{2}$ are two particular solutions of equation 2.39 it can be shown by direct differentiation that

$$
\frac{\mathrm{d}}{\mathrm{dz}}\left(\frac{\phi_{2} \phi_{1}^{\prime}-\phi_{1} \phi_{2}^{\prime}}{\mu}\right)=0
$$

Now let $\phi_{1}$ represent the wave incident from the left and $\phi_{2}$ represent the wave incident from the right which we have described above. The value of the expression within the braces in the above equation may then be evaluated both to the left and to the right of the inhomogeneity, and these two numbers equated. The two expressions are equal because, by the above equation, the quantity within the braces is independent of $z$. In this manner we obtain the equation

$$
\frac{T}{z_{2}}=\frac{\hbar}{z_{1}}
$$

This is true even if $\mathrm{k}$ is complex, representing a lossy layer. We now return to equation 2.38 and point out a few properties which will be of use in the next section also, and that make its integration a little easier in some cases. 
In equation 2.38 let us make the substitution

$$
R_{a}=e^{-2 i \int_{0}^{a} k(z) d z} D(a)
$$

and obtain

$$
\frac{d D(a)}{d a}=-\frac{Z^{\prime}(a)}{2 Z(a)}\left(e^{2 i \int_{0}^{a} k(z) d z}-e^{-2 i \int_{0}^{a} k(z) d z} D^{2}(a)\right)
$$

From this equation it is clear that, even if $k$ is a function of position, if $\mathrm{Z}$ is a constant throughout the inhomogeneity there will be no reflected wave.

But equation 2.44 is also useful in its own right, in that for high frequencies $D(a)$ will vary much less rapidly than $R_{a}$, so in any numerical integration scheme larger increments can be taken, reducing the computation time required for any particular constitutive parameter profile.

If, in equation 2.44 , we substitute $D(a)=e^{-A(a)}$, we obtain another useful form:

$$
\frac{d A(a)}{d a}=\frac{Z^{\prime}(a)}{Z(a)} \sinh \left(A(a)+2 i \int_{0}^{a} k(z) d z\right) .
$$

There are many other transformations and substitutions that have been used in obtaining equations equivalent to 2.38 (10). In concluding this section we merely make note of two which are analogous to two which will prove most useful in the next section. These are the substitutions

$$
R=\frac{i k-x}{i k+x}
$$


and

$$
R=\frac{i k Y-1}{i k Y+1}
$$

from which we obtain

$$
\begin{aligned}
& X^{\prime}+k^{2}+X^{2}-\frac{\mu^{\prime}}{\mu} X=0 \\
& Y^{\prime}-k^{2} Y^{2}-1+\frac{\mu^{\prime}}{\mu} Y=0
\end{aligned}
$$




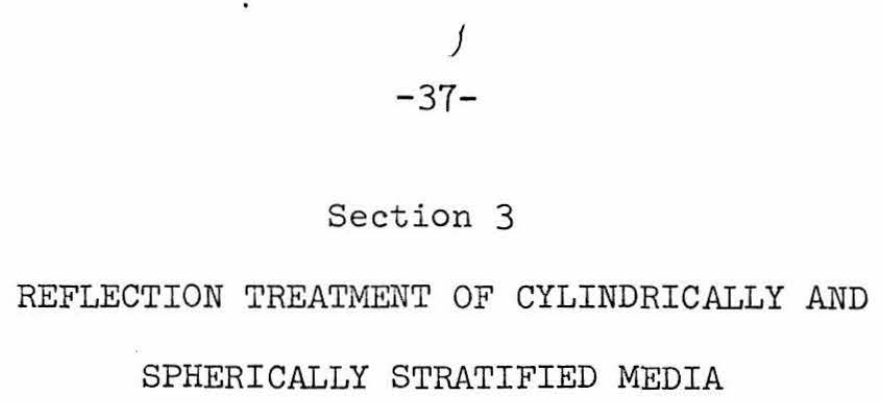

We return now to our discussion of scattering by axially symmetric inhomogeneities. Our intention is to apply to this problem the method of invariant imbedding, whose usefulness in the onedimensional case was brought out in Section 2C. It is not clear at the outset that it is feasible to use this method. However, if we allow ourselves to be guided by the application of the method to the one-dimensional scattering problem it turns out that our intention may be carried out in a fairly straightforward manner, although the algebra involved becomes cumbersome at times.

The configuration in which we are interested is that depicted in Figure IA. That is to say, a wave in which the only component of the electric field is that parallel to the axis of the cylinder is incident on an axially symmetric inhomogeneity. It was seen in Section $2 \mathrm{~A}$ that the case in which the magnetic field was parallel to the axis may be obtained from simple transformations in the formulas which we shall derive in this section.

It should also be recalled from Section $2 \mathrm{~A}$ that it is convenient for the problem under discussion to write all fields in an angular Fourier series whose coefficients vary with radius. In other words, we write in the external region $\rho>\rho_{c}$ (see Figure lA)

$$
E(\rho, \varnothing)=\sum_{-\infty}^{\infty} E_{n}(\rho) e^{i n \varnothing}
$$


where

$$
E_{n}(p)=A_{n}^{\prime} J\left(k_{c} \rho\right)+B_{n}^{\prime} H_{n}\left(k_{c} \rho\right)
$$

Now we shall assume that any incident wave of interest to us has no singularities for $\rho<\rho_{c}$. This assumption allows us to write the incident wave in the form

$$
E^{i n c}(\rho, \phi)=\sum_{-\infty}^{\infty} A_{n} J_{n}\left(k_{c} \rho\right) e^{i n \emptyset}
$$

where the coefficients $A_{n}$ are known. For example, if the incident wave is a plane wave incident from the negative $\mathrm{x}$-axis

$$
A_{n}=(i)^{n}
$$

while if the incident wave is that due to a line source of current I and at position $\left(\rho_{0}, \pi\right)$

$$
A_{n}=-\frac{\omega \mu_{c} I}{4} H_{-n}\left(k_{c} \rho_{0}\right)
$$

Now we use the condition that the scattered field must have the form of an outwardly traveling wave as $\rho \rightarrow \infty$ to write the field for $\rho>\rho_{c}$ in the form

$$
E(\rho, \varnothing)=\sum_{-\infty}^{\infty} A_{n}\left[J_{n}\left(k_{c} \rho\right)+R_{n} H_{n}\left(k_{c} \rho\right)\right] e^{i n \varnothing}
$$

where the only unknowns are the $R_{n}$, which we will call the modal reflection coefficients.

Proceeding as in Section 2A, other forms may be derived such as 
where

$$
\mathrm{E}_{\rho \rightarrow \infty}^{\operatorname{scat}}(\rho, \varnothing) \rightarrow \sqrt{\frac{2}{\pi i k_{c} \rho}} e^{i k_{c} \rho} F(\varnothing)
$$

$$
F(\varnothing)=\sum_{-\infty}^{\infty} A_{n} R_{n} e^{i n \varnothing}
$$

Because of the linearity of the problem we may calculate the $R_{n}$ by assuming an incident wave consisting of only one angular mode. That is to say, we have to solve the basic problems in which the fields in the exterior regions are

$$
\left[J_{n}\left(k_{c} \rho\right)+R_{n} H_{n}\left(k_{c} \rho\right)\right] e^{i n \varnothing}, n=0, \pm 1, \pm 2, \cdots
$$

Now, due to the axial symmetry of the inhomogeneity, it may be readily shown that for an'incident wave of the form

$$
J_{n}\left(k_{c} \rho\right) e^{i n \varnothing}
$$

the fields inside the inhomogeneity also vary as $e^{i n \varnothing}$ so that we may neglect the angular variable in our basic problems.

It should be remembered from Section $2 \mathrm{C}$ that it was useful in the one-dimensional case to define an elementary reflection coefficient $r(x, y)$. This is our motivation for the definitions we now introduce for the cylindrical problem.

The incoming elementary modal reflection coefficient

$$
\overleftarrow{r}_{n}(b, a)
$$

is defined as the modal reflection coefficient when an $n^{\text {th }}$ mode wave in 
an infinite medium, whose parameters are those of position $b$ in the final problem, is incident upon a uniform cylinder whose parameters correspond to position a in the final problem, and is of radius "a". Similarly, we may define a transmission coefficient

$$
t_{n}(b, a)
$$

which is the coefficient of the elementary solution in the interior region of the above problem. That is to say, we have

$$
\begin{array}{ll}
E_{n}(\rho)=J_{n}(k(b) \rho)+\overleftarrow{r}_{n}(b, a) H_{n}(k(b) \rho) & \rho>a \\
E_{n}(\rho)=\vec{t}_{n}(b, a) J_{n}(k(a) \rho) & \rho<a
\end{array}
$$

Imposing the condition of continuity of the tangential $\mathrm{E}$ and $H$ fields at $\rho=a$ on the above fields, we obtain

$$
\begin{aligned}
& J_{n}(k(b) a)+\overleftarrow{r}_{n}(b, a) H_{n}^{\prime}(k(b) a)=\vec{t}_{n}(b, a) J_{n}(k(a) a) \\
& J_{n}^{\prime}(k(b) a)+\overleftarrow{r}_{n}(b, a) H_{n}^{(I) \prime}(k(b) a)=\frac{\mu(b)}{\mu(a)} \frac{k(a)}{k(b)} \vec{t}_{n}(b, a) J_{n}(k(a) a) \\
& \text { Solving these equations we obtain } \\
& \overleftarrow{r}_{n}(b, a)=\frac{Z(b) J_{n}^{\prime}(k(a) a) J_{n}(k(b) a)-Z(a) J_{n}(k(a) a) J_{n}^{\prime}(k(b) a)}{Z(a) H_{n}^{(I) '}(k(b) a) J_{n}(k(a) a)-z(b) J_{n}^{\prime}(k(a) a) H_{n}^{(l)}(k(b) a)}
\end{aligned}
$$

and 


$$
\vec{t}_{n}(b, a)=\frac{J_{n}(k(b) a)}{J_{n}(k(a) a)}+\leftarrow_{n}(b, a) \frac{H_{n}^{(l)}(k(b) a)}{J_{n}(k(a) a)}
$$

As in Section 2C, these expressions will be of most use when $\mathrm{b}=\mathrm{a}+0$ when there is a jump discontinuity in the parameters of the medium at $\rho=a$. When the medium is continuous at a, the above expressions will be primarily of interest when $b=a+\Delta$ where $\Delta \ll$ a . In this approximation, and where the medium parameters are evaluated at $a$ in the following expressions, we obtain to first order in $\Delta$.

$$
\begin{gathered}
\stackrel{\leftarrow}{r}_{n}(a+\Delta, a)=\frac{\pi k a}{2 i} \cdot \Delta \cdot\left\{\frac{\dot{z}}{z} J_{n}(k a) J_{n}^{\prime}(k a)+\dot{k}_{a}\left(J_{n}^{\prime}(k a) J_{n}^{\prime}(k a)-\right.\right. \\
\left.\left.-J_{n}^{\prime \prime}(k a) J_{n}(k a)\right)\right\}
\end{gathered}
$$

$$
\begin{gathered}
\vec{t}_{n}(a+\Delta, a)=I+\frac{\pi k a}{2 i} \Delta\left\{\frac{\dot{z}}{Z} H_{n}^{(l)}(k a) J_{n}^{\prime}(k a)+\dot{k}_{a}\left(J_{n}^{\prime}(k a) H_{n}^{(I)}(k a)\right.\right. \\
\left.\left.-J_{n}^{\prime \prime}(k a) H_{n}^{(l)}(k a)\right)\right\}
\end{gathered}
$$

In the above equations

$$
\begin{aligned}
& \dot{z}=\left[\frac{d}{d \rho} \sqrt{\frac{\mu(\rho)}{\varepsilon(\rho)}}\right]_{\rho=a} \\
& \dot{k}=\left[\frac{d}{d \rho}(\omega \sqrt{\mu(\rho) \varepsilon(\rho)})\right]_{\rho=a}
\end{aligned}
$$

and 


$$
J_{n}^{\prime}(k a)=\left[\frac{d}{d x} J_{n}(x)\right]_{x=k a}^{-42-} \text {, etc. }
$$

It should also be noted that the derivation of equations 3.8 and 3.9 consists in a straightforward first order expansion about $b=a$ of equations 3.6 and 3.7 with a few applications of the Wronskian relation,

$$
\frac{\pi x}{2 i}\left(H_{n}^{(I)}(x) \cdot J_{n}^{\prime}(x)-J_{n}^{\prime}(x) H_{n}^{(I)}(x)\right)=I
$$

In addition to the modal transmission and reflection coefficients defined above, it will be found useful in the following to define coefficients calculated by assuming a source on the axis of a homogeneous cylinder. Again the parameters of the cylinder are those of position a in the final configuration, while those of the infinite homogeneous external space are those of position $b$. That is, we assume the field is of the form

$$
\begin{array}{ll}
E_{n}(\rho)=H_{n}^{(l)}(k(a) \rho)+\vec{r}_{n}(b, a) J_{n}(k(a) \rho), & \rho<a \\
E_{n}(\rho)=t_{n}(b, a) H_{n}^{(l)}(k(b) \rho), & \rho>a
\end{array}
$$

The usual boundary conditions may now be applied to obtain

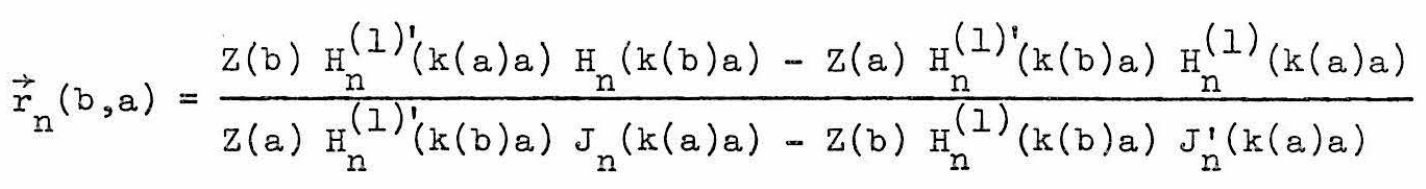

and 


$$
\overleftarrow{t}_{n}(b, a)=\frac{H_{n}(k(a) a)}{H_{n}(k(b) a)}+r_{n}(b, a) \frac{J_{n}(k(a) a)}{H_{n}(k(a) a)}
$$

Again, these expressions may be expanded about $b=a$ to first order in the forms

$$
\begin{aligned}
& \vec{r}_{n}(a+\Delta, a)=\frac{\pi k a}{2 i} \cdot \Delta \cdot\left\{\frac{\dot{z}}{Z} H_{n}^{(l)}{ }^{\prime}(k a) H_{n}^{(l)}(k a)+\dot{k}_{a}\left(H_{n}(l)^{\prime}{ }^{\prime}(k a) H_{n}(l)^{\prime}{ }^{\prime}(k a)\right.\right.
\end{aligned}
$$

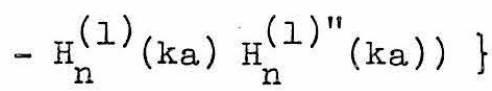

$$
\begin{aligned}
& \overleftarrow{t}_{n}(a+\Delta, a)=I+\frac{\pi k a}{2 i} \cdot \Delta \cdot\left\{\frac{\dot{z}}{Z} J_{n}(k a) H_{n}{ }^{(I)^{\prime}}(k a)+\dot{k}_{a}\left(H_{n}{ }^{(I)^{\prime}}(k a) J_{n}^{\prime}(k a)\right.\right. \\
& \left.\left.-J_{n}(k a) H_{n}^{(l) "}(k a)\right)\right\}
\end{aligned}
$$

Bearing in mind the above relations we now return to the calculation of the $R_{n}$ of equation 3.5 by considering the change in $R_{n}$ as a small additional layer is adked to the cylinder consisting of the portion of the cylinder we are interested in which lies inside the radius $\rho=a$. The situation is diagrammed in Figure 5. The small additional layer is assumed to be uniform and to have the parameters corresponding to radius $a$, while for $\rho>a+\Delta$, the parameters are those of position $a+\Delta$. We write these intermediate values of the reflection coefficient in the form $R_{n}(x)$ where $x$ is the size of the intermediate cylinder. Our object is to calculate $R_{n}=R_{n}\left(\rho_{c}+0\right)$. In other words if, for $\rho>a+\Delta$, we write the field in the form 


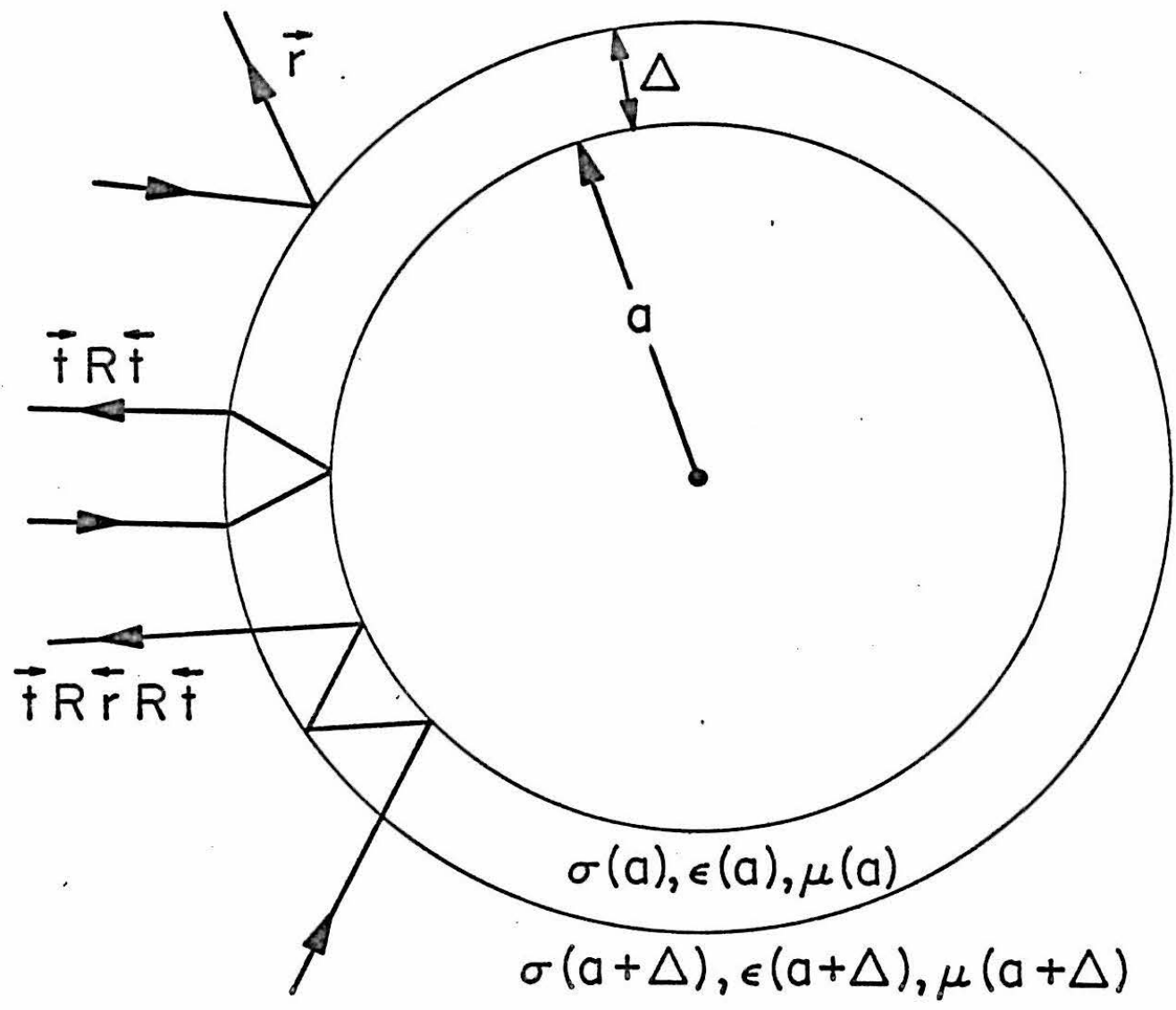

Fig. 5. Notation used in the invariant imbedding treatment of cylindrical inhomogeneities using the method of multiple reflections 


$$
E_{n}(\rho)=J_{n}(k(a+\Delta) \rho)+R_{n}(a+\Delta) H_{n}(k(a+\Delta) \rho)
$$

then by using a method of multiple reflections appropriate for cylindrical coordinates and employing the elementary reflection coefficients we have previously defined, it is clear that

$$
\begin{aligned}
R_{n}(a+\Delta) & =\overleftarrow{r}_{n}(a+\Delta, a)+\vec{t}_{n}(a+\Delta, a) R_{n}(a) \overleftarrow{t}_{n}(a+\Delta, a) \\
& +\vec{t}_{n}(a+\Delta, a) R_{n}(a) \vec{r}_{n}(a+\Delta, a) R_{n}(a) \overleftarrow{t}_{n}(a+\Delta, a)+\cdots
\end{aligned}
$$

Summing this series we may write

$$
R_{n}(a+\Delta)=\overleftarrow{r}_{n}(a+\Delta, a)+\frac{\vec{t}_{n}(a+\Delta, a) \overleftarrow{t}_{n}(a+\Delta, a) R_{n}(a)}{1-R(a) \vec{r}_{n}(a+\Delta, a)}
$$

However, for the moment we stick with expression 3.14 which we expand for small $\Delta$, using equations $3.8,3.9,3.12$ and 3.13 in the form

$$
\begin{aligned}
R_{n}(a)+ & \Delta \dot{R}_{n}(a)=\frac{\pi k a}{2 i} \cdot \Delta \cdot\left\{\frac{\dot{Z}}{Z} J_{n}(k a) J_{n}^{\prime}(k a)+k a\left(J_{n}^{\prime}(k a) J_{n}^{\prime}(k a)\right.\right. \\
& \left.\left.-J_{n}^{\prime \prime}(k a) J_{n}(k a)\right\}\right\}+R(a) \\
+ & R(a) \frac{\pi k a}{2 i} \Delta\left\{\dot{k}_{a} J_{n}^{\prime}(k a) H_{n}^{\prime}(k a)+\frac{\dot{Z}}{Z}\left(J_{n}(k a) H_{n}^{\prime}(k a)\right.\right. \\
+ & \left.\left.H_{n}(k a) J_{n}^{\prime}(k a)\right)-\dot{k a}_{n}\left(J_{n}(k a) H_{n}^{\prime \prime}(k a)+H_{n}(k a) J_{n}^{\prime \prime}(k a)\right)\right\} \\
& +R_{n}^{2}(a) \frac{\pi k a}{2 i} \Delta\left\{\frac{\dot{Z}}{Z} H_{n}(k a) H_{n}^{\prime}(k a)+\dot{k}_{a}\left(H_{n}^{\prime}(k a) H_{n}^{\prime}(k a)\right.\right. \\
& \left.-H_{n}(k a) H_{n}^{\prime \prime}(k a)\right\}+0\left(\Delta^{2}\right)
\end{aligned}
$$

In writing the above equation we have implicitly assumed that the medium 
is continuous at $\rho=a$ by using $3.8,3.9,3.12,3.13$. If this is not true we may, for a step discontinuity in the medium, write a "jump conaition" in the form:

$$
R_{n}(a+0)=\overleftarrow{r}_{n}(a+0, a)+\frac{\vec{t}_{n}(a+0, a) \overleftarrow{t}_{n}(a+0, a) R_{n}(a)}{I-R_{n}(a) \vec{r}_{n}(a+0, a)}
$$

However, for regions in which the medium is continuous we assume the validity of equation 3.16 , divide it by $\Delta$ and allow $\Delta \rightarrow 0$, obtaining

$$
\begin{aligned}
\frac{2 i}{\pi} \dot{R}_{n}(a)= & k a\left\{\left[\frac{\dot{Z}}{Z} J_{n}(k a) J_{n}^{\prime}(k a)+\dot{k} a\left(J_{n}^{\prime}(k a) J_{n}^{\prime}(k a)-J_{n}(k a) J_{n}^{\prime \prime}(k a)\right)\right]\right. \\
& \left.+R_{n}^{2}(a)\left[\frac{\dot{Z}}{Z} H_{n}(k a) H_{n}^{\prime}(k a)+\dot{k}_{a}\left(H_{n}^{\prime}(k a) H_{n}^{\prime}(k a)-H_{n}(k a) H_{n}^{\prime \prime}(k a)\right)\right]\right\} \\
& +k a R_{n}(a)\left\{\frac{\dot{Z}}{Z}\left(J_{n}(k a) H_{n}^{\prime}(k a)+H_{n}(k a) J_{n}^{\prime}(k a)\right)\right. \\
& \left.+\dot{k}_{a}\left(2 H_{n}^{\prime}(k a) J_{n}^{\prime}(k a)-J_{n}(k a) H_{n}^{\prime \prime}(k a)-H_{n}(k a) J_{n}^{\prime \prime}(k a)\right)\right\}
\end{aligned}
$$

The above equation may be simplified somewhat. Assuming the argument of all Bessel functions in the following equation to be ka, equation 3.18 may be manipulated into the form

$$
\begin{aligned}
\frac{2 i}{\pi k a} \frac{d R_{n}(a)}{d a} & =\dot{k} a\left\{\left(J_{n}^{\prime}+R_{n}(a) H_{n}^{\prime}\right)^{2}-\left(J_{n}+R_{n}(a) H_{n}\right)\left(J_{n}^{\prime \prime}+R_{n}(a) H_{n}^{\prime \prime}\right)\right\} \\
& +\frac{\dot{z}}{z}\left(J_{n}+R_{n}(a) H_{n}\right)\left(J_{n}^{\prime}+R_{n}(a) H_{n}^{\prime}\right)
\end{aligned}
$$

Although we need further development to attain a practical method of calculation, it can be seen that in principle we have already accomplished our object. That is to say, we may integrate equation 
3.19 numerically if we so desire, using equation 3.17 at any points of discontinuity of the medium. In employing equation 3.17 we must use the definitions $3.8,3.9,3.12$ and 3.13 . The integration of 3.19 starts at $a=0$ with

$$
R_{n}(0)=0
$$

and proceeds to $a=\rho_{c}$ at which

$$
R_{n}=R_{n}\left(\rho_{c}+0\right)
$$

The coefficients calculated according to equation 3.20 may then be substituted in equations such as 3.4 to calculate various field quantities of interest.

If a table of $R_{n}(a)$ is kept as the integration of equation 3.19 proceeds, it can be seen that we have information as to the fields in the external region which pertains to a whole class of inhomogeneous cylinders.

However, it is inadvisable to stop at equation 3.19 , as that equation is extremely cumbersome and a lot of computer time would be wasted if it were used directly. But before any further algebra is indulged in, there are a couple of interesting physical facts which may be brought out by studying equation 3.19 directly.

If the impedance of the medium does not vary with position, i.e. if

$$
z(a)=z\left(\rho_{c}\right) \text { or } \dot{Z}(a)=0
$$

then equation 3.19 becomes 


$$
\frac{2 i}{\pi k a} \dot{R}_{n}=\dot{k a}\left\{\left(J_{n}^{\prime}+R_{n} H_{n}^{\prime}\right)^{2}-\left(J_{n}+R_{n} H_{n}\right)\left(J_{n}^{\prime \prime}+R_{n} H_{n}^{\prime \prime}\right)\right\}
$$

From this equation it can be seen that even though $\mathrm{Z}$ does not vary with position, there is, in general, a reflected wave, unlike the one-dimensional case discussed in Section 2C.

However, there is one interesting fact associated with the constant impedance case. We recall that the $\mathrm{H}$ polarization case may be handled in a manner precisely analogous to that given so far in this section. The only change will be to interchange $\varepsilon(\rho)$ and $\mu(\rho)$. It is obvious that this interchange has no effect on equation 3.21. This means, for example, that for the constant impedance case the differential scattering width of the inhomogeneous cylinder for a wave incident perpendicular to the cylinder axis is independent of the polarization of the incident wave.

In any case we see that the $\mathrm{H}$ polarization problem may be handled by replacing $\dot{z} / z$ by $-\dot{Z} / z$ on the right hand side of equation 3.19 . From this it is clear that if we change polarizations and at the same time interchange the dependence of $\varepsilon$ and $\mu$ on the radius, the scattering pattern remains the same.

There is one other special case of 3.19 which may be of interest, that is, the one in which one of $\varepsilon, \mu$ is independent of position. For example if we have $E$ polarization and $\mu$ constant, 3.19 becomes

$$
\frac{2 i}{\pi k a} \frac{d R_{n}(a)}{d a}=\dot{k} a\left\{\left(J_{n}^{\prime}+R_{n} H_{n}^{\prime}\right)^{2}-\left(J_{n}+R_{n} H_{n}\right)\left(J_{n}^{\prime \prime}-R_{n} H_{n}^{\prime \prime}\right)-\frac{\left(J_{n}+R H_{n}\right)\left(J_{n}^{\prime}+R H_{n}^{\prime}\right)}{k a}\right\}
$$


in which the explicit dependence on $\mathrm{Z}$ drops out.

It should be noted that the forms we have obtained so far of the rate-of-change equation for the modal reflection coefficients are analogous to equation 2.44 in that they are suitable for numerical integration even for high values of $k$ as long as the derivative of the parameters with radius is of reasonable size. It is clear that this is true from an observation that the right hand side of equation 3.19 reduces to zero in a constant parameter region. This result has been achieved directly by means of the original definition of $R_{m}$ implicit in expression 3.5 A definition more in line with the work of Section $2 \mathrm{C}$ would be to write the field for $\rho>a>\rho_{c}$ in the form

$$
\left[\frac{J_{n}(k(a) \rho)}{J_{n}(k(a) a)}+\frac{R_{n}^{*} H_{n}^{(l)}(k(a) \rho)}{H_{n}^{(I)}(k(a) a)}\right] e^{i n \phi}
$$

The calculation could then be carried through to obtain an equation analogous to equation 3.19 for $R_{n}^{*}$. A subsequent transformation removing the rapid variation of $R_{n}^{*}$ could bring us back to 3.19 , but this has all been avoided. There is one other difference with the one dimensional case which should be brought out. We have, in the cylindrical case, what is essentially a standing wave incident on the inhomogeneity, while in the one-dimensional case we had a traveling wave。

The two differences mentioned above in conjunction with the asymptotic form of expression 3.5 


$$
\sqrt{\frac{2}{\pi k_{c} \rho}\left[e^{i\left(k_{c} \rho-\frac{n \pi}{2}-\frac{\pi}{4}\right)}+e^{-i\left(k_{c} \rho \frac{n \pi}{2}-\frac{\pi}{4}\right)}+R_{n} e^{i\left(k_{c} \rho-\frac{n \pi}{2}-\frac{\pi}{4}\right)}\right]} \underbrace{2}_{\rho \rightarrow \infty}
$$

lead to the conjecture that there might be some correspondence between r $R_{n}$ in the cylindrical case and

$$
\frac{1}{2}\left(e^{-2 i\left(k_{c} \rho-\frac{n \pi}{2}-\frac{\pi}{4}\right)} R-1\right)
$$

where $R$ is some one-dimensional reflection coefficient defined by some equation such as 2.38 .

We now examine this conjecture in more detail. First we note that for $\dot{k}>-k / a$, the arguments of the Bessel functions in equation 3.19, namely $\mathrm{k}(\mathrm{a}) \mathrm{a}$, are monotonically increasing functions of $\mathrm{a}$. If $\mathrm{k}$ is fairly large then, beyond a certain radius the Bessel functions may be replaced by their asymptotic forms

$$
\begin{aligned}
& J_{n}(x) \rightarrow \sqrt{\frac{2}{\pi x}} \cos \left(x-\frac{n \pi}{2}-\frac{\pi}{4}\right) \\
& H_{n}(x) \rightarrow \sqrt{\frac{2}{\pi x}} e^{i\left(x-\frac{n \pi}{2}-\frac{\pi}{4}\right)}
\end{aligned}
$$

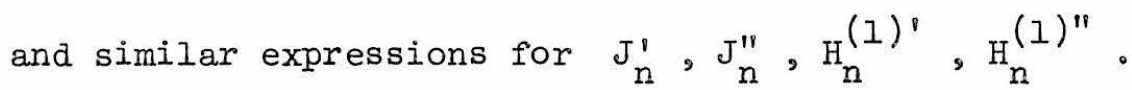

Substituting all these in equation 3.19 we arrive at the equation 


$$
\begin{aligned}
\frac{2 i}{\pi k a} \dot{R}_{n} & =\dot{k} a\left\{\frac{-2}{\pi k a} e^{2 i \alpha_{n}}\left(\frac{1-e^{-2 i \alpha_{n}}}{2}+\left.R_{n}\right|^{2}+\frac{2}{\pi k a} e^{2 i \alpha_{n}}\left(\frac{1+e^{-2 i \alpha_{n}}}{2}+R_{n}\right)^{2}\right\}\right. \\
& +\frac{\dot{z}}{z} \cdot \frac{2 i}{\pi k a} e^{2 i \alpha_{n}}\left(\frac{1+e^{-2 i \alpha_{n}}}{2}+R_{n}\right)\left(\frac{1-e^{-2 i \alpha_{n}}}{2}+R_{n}\right)
\end{aligned}
$$

where

$$
\alpha_{n}=k(a) a-\frac{n \pi}{2}-\frac{\pi}{4}
$$

Now introducing the substitution suggested by expression 3.22

$$
\frac{1}{2}+R_{n}(a)=\frac{1}{2} e^{-2 i \alpha_{n}} u_{n}(a)
$$

and simplifying, the asymptotic form of the equation becomes

$$
\dot{u}_{n}=2 i k u_{n}-\frac{\dot{z}}{2 z}\left(1-u_{n}^{2}\right)
$$

which is seen to be identical in form to equation 2.38 . The equation itself is independent of $n$ and the $u_{n}(a)$ functions differ for different values of $n$ only because their initial values at some $u_{n}\left(a_{0}\right)$ are not the same.

Now it is known from the theory of the Riccati equation that if we have three particular solutions of equation 3.24 , say $X(a), Y(a)$ and $\mathrm{Z}(\mathrm{a})$, then the general solution may be written in the form

$$
u_{n}(a)=\frac{Y(X-Z)-C_{n} Z(X-Y)}{(X-Z)-C_{n}(X-Y)}
$$

where $C_{n}$ is some arbitrary constant which is to be determined by the value of $u_{n}(a)$ at some starting point. 
For example, suppose it is decided that the asymptotic form of the equations is sufficiently accurate for $a>a_{0}$. Then we only need three particular solutions of 3.24 for $a>a_{0}$, say those for which

$$
\begin{aligned}
& X\left(a_{0}\right)=0 \\
& Y\left(a_{0}\right)=1 \\
& Z\left(a_{0}\right)=-1
\end{aligned}
$$

then it is clear from a substitution in 3.25 that

$$
c_{n}=\frac{I-u_{n}\left(a_{0}\right)}{1+u_{n}\left(a_{0}\right)}
$$

Our program would then be to use 3.19 to calculate the $R_{n}$ (a) out to $a_{0}$, then to use $3.23,3.26$ and 3.25 in turn, to calculate $u_{n}(a)$ for any $a>a_{0}$ for which we have calculated $X(a), Y(a)$ and $Z(a)$. Employing equation 3.23 a second time will then give us $R_{n}(a)$ for all relevant values of $\mathrm{n}$ with much less computational effort than using 3.19. for all $n$ and $a$. It is to be understood here that we have in mind a reduction of calculation time in a digital computer.

There are, of course, other means of reducing computational time and of further reducing the time required for the above scheme. We wish to discuss here two in particular. One involves manipulating the right hand side of equation 3.19 into forms which are more easily evaluated numerically without changing the basic equation. The second method of reducing calculation is to make a transformation of the independent or the dependent variables in equation 3.19 so that it assumes 
a simpler form. An example of this is in the above transformations to $u_{n}$ for the asymptotic form of the equation, but there are also useful changes to be made directly in 3.19 .

First, however, we discuss a few of the possible manipulations of the Bessel functions in the rate of change equation. The purpose of these transformations is to cast equation 3.19 into the form for which numerical evaluation of the right hand side is easiest. There are two things to keep in mind in trying to attain a fast computational form. The first is that the Bessel functions are most easily obtained using their difference equation:

$$
J_{n-1}(x)=\frac{2 n}{x} J_{n}(x)-J_{n-1}(x)
$$

and the second is that the derivatives of Bessel functions in equation 3.19 may be replaced by functions of different order using

$$
J_{n}(x)=\frac{1}{2}\left(J_{n-1}(x)-J_{n+1}(x)\right)
$$

From these facts it is clear that the form we desire is that in which no derivatives of Bessel functions occur, but any number of orders may occur. This is clear because in calculating the Bessel functions we have to calculate many orders in any case. The easiest way to state the final form of these transformations is to rewrite equation 3.19 as

$$
\begin{aligned}
& \frac{2 i}{\pi} R=k a\left\{\left[\frac{\dot{Z}}{Z} J_{n} J_{n}^{\prime}\right.\right.\left.+\dot{k}_{a}\left(J_{n}^{\prime} J_{n}^{\prime}-J_{n}^{\prime \prime} J_{n}\right)\right] \\
&\left.+R^{2}\left[\frac{\dot{Z}}{Z} H_{n}^{H_{n}^{\prime}}+\dot{k}_{a}\left(H_{n}^{\prime} H_{n}^{\prime}-H_{n} H_{n}^{\prime \prime}\right)\right]\right\} \\
&+\operatorname{ka} R\left\{\frac{\dot{Z}}{Z}\left(J_{n} H_{n}^{\prime}+H_{n} J_{n}^{\prime}\right)+\dot{k}\left(2 J_{n}^{\prime} H_{n}^{\prime}-J_{n} H_{n}^{\prime \prime}-H_{n} J_{n}^{\prime \prime}\right)\right\}
\end{aligned}
$$


and then note the equivalent forms

$$
\begin{aligned}
J_{n} J_{n}^{\prime} & =J_{n-1} J_{n}-\frac{2 n}{x} J_{n}^{2} . \\
J_{n}^{\prime} J_{n}^{\prime}-J_{n}^{\prime \prime} J_{n} & =\left(J_{n-1}-\frac{2 n}{x} J_{n}\right)\left(J_{n-1}-\frac{2 n-1}{x} J_{n}\right)+J_{n}^{2}\left(\frac{1-n^{2}}{x^{2}}\right)
\end{aligned}
$$

and two similar expressions with $H_{n}$ replacing $J_{n}$, and the equation

$$
\begin{aligned}
2 J_{n}^{\prime} H_{n}^{\prime}-J_{n} H_{n}^{\prime \prime}-H_{n} J_{n}^{\prime \prime} & =2 J_{n-1} H_{n-1}+\left(I-\frac{4 n}{x}\right)\left(J_{n} H_{n-1}+J_{n-1} H_{n}\right) \\
& +\left(2-\frac{4 n}{x}+\frac{6 n^{2}}{x^{2}}\right) J_{n} H_{n} .
\end{aligned}
$$

Of course many other forms of the above equations may be easily written down, but the above expressions involve the least computation that one can achieve without any transformation of variables in equation 3.19.

We now turn to the last subject of the present section involving a cylindrical inhomogeneity, i.e. that of simplifying equation 3.19 by transforming the variables.

We define a new variable $\phi(a)$ by the equation

$$
R_{n}(a)=\frac{\emptyset_{n}(a) J_{n}(k a)-J_{n}^{\prime}(k a)}{H_{n}^{\prime}(k a)-\phi_{n} H_{n}(k a)}
$$

from which we may calculate the expressions

$$
J(k a)+R_{n}(a) H_{n}(k a)=\frac{2 i}{\pi k a} \frac{1}{H_{n}(k a)-\phi_{n}(a) H_{n}(k a)}
$$




$$
\begin{aligned}
J_{n}^{\prime}(k a)+R_{n}(a) H_{n}^{\prime}(k a) & =\frac{2 i}{\pi k a} \frac{\phi_{n}(a)}{H_{n}^{\prime}(k a)-\phi_{n}(a) H_{n}(k a)} \\
J_{n}^{\prime \prime}(k a)+R_{n}(a) H_{n}^{\prime \prime}(k a) & =\left(J_{n}^{\prime \prime} H_{n}^{\prime}-J_{n}^{\prime} H_{n}^{\prime \prime}\right)+\emptyset_{n}(a)\left(H_{n}^{\prime \prime} J_{n}-J_{n}^{\prime \prime} H_{n}\right) \\
& =\frac{1-n^{2}}{(k a)^{2}} \frac{2 i}{\pi k a}+\phi_{n}(a) \frac{2 i}{\pi(k a)^{2}}
\end{aligned}
$$

Substituting all these in equation 3.19 and calculating the derivative on the left hand side in terms of $\varnothing$ we obtain

$$
\dot{\phi}_{n}=\phi_{n}\left(\frac{\dot{z}}{z}-\frac{1}{a}\right)-k\left(I+\phi_{n}^{2}\right)+\frac{n^{2}}{|k a|^{2}} k^{*}
$$

In this equation all the dependence on Bessel functions has been eliminated. Equation 3.28 is analogous to equation 2.38 in that neither contains the "natural" variation of the dependent variable. That is to say, even in a constant parameter, region $\varnothing$ may change rapidly, whereas $\mathrm{R}$ will not change. In spite of this drawback, however, equation 3.28 is to be preferred to equation 3.19 when the inhomogeneity is small enough; that is, when $\rho_{c} / \lambda_{c}$ is fairly small (less than 2 or 3), simply because all the calculation of Bessel functions has been avoided.

In using equation 3.28 one should be a little careful. Although for physical reasons $R_{n}$ will be bounded, there is no such guarantee for $\phi_{n}$. In fact $\phi_{n}$ can become very large at many discrete points. This difficulty can be overcome, however, by considering a second variable, in addition to $\emptyset_{n}$, 


$$
\psi_{\mathrm{n}}=I / \phi_{\mathrm{n}}
$$

whose equation is

$$
\dot{\psi}_{n}=\psi_{n}\left(\frac{1}{a}-\frac{\dot{z}}{z}\right)+k\left(1+\psi_{n}^{2}\right)-\frac{n^{2} \psi_{n}^{2}}{|k a|^{2}} k * .
$$

In our numerical calculation we integrate equation 3.28 or 3.29 , simply choosing the smallest of $\phi_{n}$ and $\psi_{n}$ as our dependent variable. A switch from one to the other may be carried out at any intermediate point in the integration for which our variable becomes greater than unity.

There are two other minor points which are evident from a closer examination of equation 3.28 and 3.29. The first of these is that the starting values which must be chosen so that $R_{n}(0)$ is zero are

$$
\left.\begin{array}{l}
\phi_{0}(a) \rightarrow-\frac{k a}{2} \\
\psi_{n}(a) \rightarrow \frac{k a}{n-1} n>1
\end{array}\right\} \quad \text { as } a \rightarrow 0
$$

The second point to remember is that in integrating across a discontinuity in the medium we must use "jump conditions" which evidently may be written in the form

$$
\left.\begin{array}{l}
\phi_{n}(a+0)=\frac{Z(a+0)}{Z(a-0)} \phi_{n}(a-0) \\
\psi_{n}(a+0)=\frac{Z(a-0)}{Z(a+0)} \psi_{n}(a-0)
\end{array}\right\}
$$

A computer code was written, based on the above scheme of calculation. The details of the code appear in an appendix, while some 
of the results obtained with it are described in the fifth section.

The remainder of the present section is devoted to a brief summary of the results for spherical inhomogeneities.

For the treatment of spherically symmetric inhomogeneities we first represent the incident waves in terms of their multipole expansion. For example, for an incident plane wave traveling in the positive $z$ direction and which is linearly polarized, it is possible to write (7)

$$
\begin{aligned}
& \underline{E}_{i}=e_{x} E_{0} e^{i k z}=E_{0} \sum_{n=1}^{\infty} i^{n} \frac{2 n+l}{n(n+1)}\left(\underline{m}_{0 l n}^{(I)}-i \underline{n}_{e l n}^{(I)}\right) \\
& \underline{H}_{i}=e_{y} \frac{E_{0}}{Z_{c}} e^{i k z}=-\frac{E_{0}}{Z} \sum_{n=1}^{\infty} i^{n} \frac{2 n+1}{n(n+1)}\left(\underline{m}_{e l n}^{(I)}+i \underline{n}_{0 l n}^{(I)}\right)
\end{aligned}
$$

where

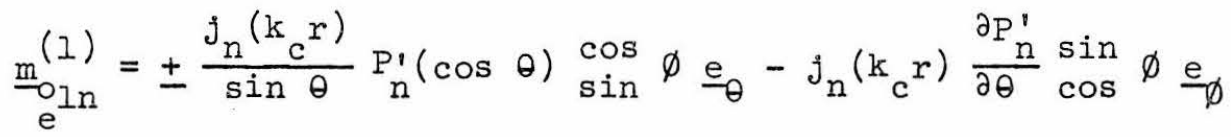

$$
\begin{aligned}
& \left.\left.\underset{\mathrm{e}^{\mathrm{m}}}{(1)}=\frac{\mathrm{n}(\mathrm{n}+1)}{\mathrm{k}_{c} r} j_{n}\left(k_{c} r\right) P_{n}^{\prime}(\cos \theta) \cos _{\cos }^{\sin } \phi \underline{e}_{r}+\frac{1}{k_{c} r}\left[k_{c} r j_{n}\right) k_{c} r\right)\right]^{\prime} \\
& \times \frac{\partial p_{n}^{\prime}}{\partial \theta} \sin \phi \underline{e}_{\theta} \pm \frac{1}{k_{c} r \sin \theta}\left[k_{c} r j_{n}\left(k_{c} r\right)\right] \cdot P_{n}^{\prime}(\cos \theta) \\
& \times \frac{\cos }{\sin } \varnothing e_{\phi}
\end{aligned}
$$

Now each multipole has either a zero radial electric field or a zero radial magnetic field ( $E$ or $H$ ). The $E$ fields, for example, have 


$$
\begin{aligned}
& \underset{\mathrm{e}^{\mathrm{mn}}}{\mathrm{H}^{\mathrm{E}}}=-i \mathrm{H} \underset{\mathrm{e}_{\mathrm{mn}}^{\mathrm{m}}}{(\mathrm{I})}
\end{aligned}
$$

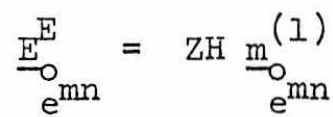

From these and the representations of the incident field it is clear that it is only necessary to calculate the reflection coefficients of each elementary multipole wave. This is most easily done by considering the tangential fields rather than the radial fields which are usually of prime importance when discussing multipole expansions.

For example we consider the $E_{m n}$ mode. By writing, say, $H_{\theta}$ in the external region in the form

$$
F_{n m}(\theta, \varnothing)\left[j_{n}(k r)+R_{n}^{E} h_{n}(k r)\right]
$$

it follows from a step by step analogy with the calculations for the cylinder, using the continuity of the tangential component of $E$ at each boundary and the equation

$$
\underline{E}=\frac{\nabla \times \underline{H}}{-i \omega \varepsilon}
$$

that:

$$
\begin{aligned}
-\frac{i}{k a} \dot{R}_{n}^{E}(a) & =\left\{\frac{\dot{\varepsilon}}{\varepsilon} j_{n}(k a)\left[k a j_{n}(k a)\right]^{\prime}+\dot{k}_{a}\left[k a\left(j_{n}^{\prime} j_{n}^{\prime}-j_{n} j_{n}^{\prime \prime}\right)-j_{n} j_{n}^{\prime}\right]\right\} \\
+ & R_{n}^{E}\left\{\frac{\dot{\varepsilon}}{\varepsilon}\left[h_{n}\left(k a j_{n}\right)^{\prime}+j_{n}\left(k a h_{n}\right)^{\prime}\right]+\dot{k}\left[k a\left(j_{n}^{\prime} h_{n}^{\prime}-j_{n} h_{n}^{\prime \prime}\right)-j_{n} h_{n}^{\prime}\right]\right. \\
& \left.+\dot{k} a\left[k a\left(h_{n}^{\prime} j_{n}^{\prime}-h_{n} j_{n}^{\prime \prime}\right)-h_{n} j_{n}^{\prime}\right]\right\} \\
& +R_{n}^{E^{2}}\left\{\frac{\dot{\varepsilon}}{\varepsilon} h_{n}\left[k a h_{n}\right] \prime+k a\left[k a\left(h_{n}^{\prime} h_{n}^{\prime}-h_{n} h_{n}^{\prime \prime}\right)-h_{n} h_{n}^{\prime}\right]\right\}
\end{aligned}
$$


where the dot denotes differentiation with respect to a while the prime indicates differentiation with respect to ka. By some manipulation it is possible to write the above equation in the simplified form, quite similar to 3.19

$$
\begin{aligned}
\dot{\mathrm{R}}_{n}^{E}= & \dot{\mathrm{k} a}\left\{(\mathrm{ka})^{2}\left[\left(j_{n}^{\prime}+\mathrm{R}_{n}^{E} \mathrm{~h}_{n}^{\prime}\right)^{2}-\left(j_{n}+R_{n}^{E} h_{n}\right)\left(j_{n}^{\prime \prime}+R_{n}^{E} h_{n}^{\prime \prime}\right)\right]\right. \\
& \left.+\left(j_{n}+R_{n}^{E} h_{n}\right)^{2}\right\}-\frac{\dot{z}}{z}\left(j_{n}+R_{n}^{E} h_{n}\right)\left(j_{n}^{\prime}+R_{n}^{E} h_{n}^{\prime}\right)(k a)^{2}
\end{aligned}
$$

From this it is clear that the equation for $\mathrm{R}_{\mathrm{n}}^{\mathrm{H}}$ is exactly the same as the one above with $-\dot{Z} / Z$ replaced by $\dot{Z} / Z$. It also follows from the above equation that if $\mu / \varepsilon=\mu_{c} / \varepsilon_{c}$ then the $E$ and $H$ modes will have exactly the same reflection coefficient. When this fact is used, together with equation 3.31 and the limiting form of equation 3.32 as $\theta \rightarrow \pi$, a short calculation suffices to convince one that the scattered field in the direction opposite to the incident direction is zero. The fact that the back-scattering cross-section of a sphere with constant $\mu / \varepsilon$ is zero was proved recently by another method (8).

There are, of course, simplifications and transformations of equation 3.33 that could be demonstrated. However, it is felt that these, for the most part, would not be too instructive, since almost all of them have analogies which have already been discussed in connection with the cylindrical case.

Further geometries are briefly discussed in the conclusion of this report. 


\section{Section 4}

\section{SURVEY AND COMPARISONS}

The object of the present section is to look back over the fairly messy computations of the last section and observe what really has been accomplished. An additional purpose is to compare the method of Section 3, in various aspects, with those discussed in the second section.

The first point that should be made is that what has been derived in the last section is primarily a practical computational method. There were certain statements of a general or theoretical nature that we were able to make from observations of the various forms of the rate-of-change equation, but these were of secondary importance. For example, to take the most recent case, although it is interesting that the radar cross-section of a spherically symmetric object with constant "impedance" is zero, it is not really very important. However, a means of rapidly calculating the cross section of an arbitrary spherically or cylindrically symmetric object is of practical importance for certain studies of present interest such as reflection from a nuclear fireball or meteor trail.

It is well to realize that although many of the computations of the last section are cumbersome and ugly, the main results can be summarized in a few equations. For the cylinder these equations are the first rate-of-change equation derived, equation 3.19, along with certain of its simplifications for the far field, resulting in equations 3.23 and 3.24 . The various other forms of the rate-of-change 
equation, for example the one useful for smaller cylinders written as equations 3.28 and 3.30 , are not as important. Although these other forms are necessary in making the method practically useful, they are all just transformations of the basic equation.

Another point which should be stressed concerning the work of the last section is that, although the work is rather involved, the principles are fairly simple. We merely wish some method of calculating the change in reflection coefficient for some particular mode as our inhomogeneity receives a small additional layer. The so-called method of multiple reflections with which we obtained this equation is not the only way our object could have been attained. For example, we could solve a complete boundary value problem for reflection from a cylinder, with some arbitrary reflection coefficient, onto which is attached an additional uniform layer of finite thickness. An examination of the behavior of the total reflection coefficient of this structure as the thickness of the extra layer tended to zero would again give us the rate of change equation. It seems, however, that the method used in the last section is the most appealing from a physical standpoint. In addition, it bears the closest resemblance to methods used in previous applications of the method of invariant imbedding, such as reflection from a foggy meāium, etc.

There are some additional remarks which should be made in relating the work of the second and third sections.

As opposed to the method of separation of variables, there are two general comments which should be made in favor of the method of Section 3. The first of these is that the method of separation of 
variables results in a second order equation for the field strengths. Numerically this is intrinsically more difficult to solve than a first order equation in the sense that it takes approximately twice as much time. In addition, for slightly more complex geometries there might be some difficulty in recognizing the correct initial conditions for the field equation. For example, in elliptical geometries, and even in the circular one, the correct value for the initial first derivative is not very easy to recognize. On the other hand, the fact that there should be no reflection when the scatterer disappears is always obvious, and this is sufficient as far as initial conditions for the rate-of-change equation is concerned.

The second comment which should be made about the method of separation of variables is that it is not as easy to state the correct boundary conditions in some cases (for example when we choose a dependent variable such as $\sqrt{\mu \mathrm{E}}$ in order to simplify our differential equation, or when we are concerned with reflection from a moving medium). It is always a straightforward, although perhaps lengthy, matter, using the basic physical equations, to build the correct boundary conditions into the rate-of-change equation approach. This is shown, for example, in the moving medium case by the work in reference (9). .

The reasons for which the rate-of-change method is to be preferred to the thin-shell approximation are numerous. One is that the rate-of-change method results immediately in an explicit calculational recipe. The thin shell approximation, as it is usually applied, results in a matrix equation which must be inverted. Although, because 
of the form of this matrix there are certain simplifications which may be made resulting in an explicit calculation of the type exhibited in equation 2.31, this is essentially a two-step procedure for obtaining an approximation to the results of the one-step rate-of-change derivation.

However, there is an additional and more serious objection to be made to the procedure of approximating the inhomogeneous problem by several thin shells. This is that there is some doubt as to the correctness of the reflection coefficients calculated by such a method as the thickness of the shells approaches zero. This can be seen from an examination of the one-dimensional case and in particular, equation $2.36:$

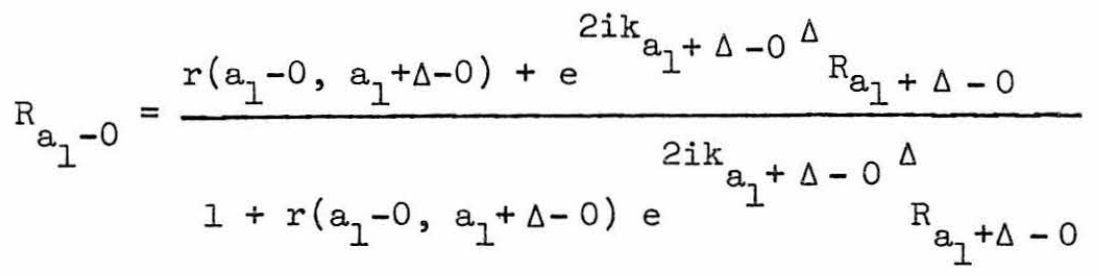

At first sight it would seem from equation 2.36 that as the layer thickness vanishes, the reflection coefficient remains constant, since

$$
r\left(a_{1}-0, a_{1}-0\right) \rightarrow 0
$$

This is, of course, not true, since what we are calculating with equation 2.36 is merely the change in reflection coefficient as our reflecting surface changes by $\Delta$. But the facts that the right hand side of equation 2.36 vanishes with vanishing $\Delta$ and that the thin shell approximation is based on equations similar to 2.36 , imply that as the shells become thinner what we are effectively doing with this method is differentiating numerically with a differentiation scheme 
based on a very small interval. The gross numerical inaccuracies of such a calculation are well known. On the other hand, for rate-ofchange equations such as equation 2.38 , the differentiation is done analytically, so this entire problem is circumvented.

Before concluding this section we would like to recall to attention the close analogy between the one-dimensional case and the cylindrically symmetric case as treated by the method of "invariant imbedding". This is especially clear when one compares the large cylinder approximation, equation 3.24 , with equation 2.38 . It seems that a similar treatment of an elliptical or other boundary can be made to yield similar simplifications.

Finally, we wish to emphasize that the method is primarily a computational method. It achieves its usefulness by restricting attention to the fields in the exterior region. In addition to being a rapid method of calculating the field scattered from a particular cylinder, the method can, with no additional effort, be used to compute the fields scattered from a whole class of similar cylinders (or spheres, or other geometrical shapes for which the rate-of-change equations can be derived).

In the next section a few examples of application of the method are exhibited. 


\section{Section 5}

EXAMPLES OF CALCULATIONS FOR THE CYLINDRICAL CASE

Using the method of Section 3, calculations have been made for three particular types of inhomogeneous cylinder with various types of incident wave. The computer program by which these calculations were made is described in the Appendix.

The first set of data with which comparisons were made was that of Jasik (1). In 1954 Jasik published the results of his calculations concerning the exact theory of the two-dimensional Luneberg lens.

A two-dimensional Luneberg lens is an axially symmetric inhomogeneous dielectric cylinder whose dielectric constant varies with radius according to the relation

$$
\varepsilon(\rho)=\sqrt{2-\rho^{2} / a^{2}} \varepsilon_{0}
$$

If the ray-optical approximation is employed to calculate the far field of a line source at the boundary of a cylinder with the above variation of dielectric constant, it is found that the far field as a function of angle has a delta-function peak in the forward direction. A closer approximation in which ray optics is used only to calculate the magnitude of the field in a plane tangent to the front edge of the cylinder, and then a Kirchhoff integral is used to calculate the far field, leads to a far field as a function of angle in the form

$$
F(\varnothing) \propto \frac{J_{3 / 4}(k a \sin \theta)}{k a \sin \theta}
$$


Jasik (1) exhibits a curve for the far field for the case when $\mathrm{ka}=8$, which is identical with Figure 6a. He discusses also the validity of the optical approximations with regard to the far fields they predict. The interesting point is that the physical optics approximation is so accurate, even at fairly low ka numbers.

Figure 6 is entirely based on computations employing the method of invariant imbedding. Jasik did not obtain any exact results for the higher ka numbers for which curves $6 \mathrm{~b}$ and $6 \mathrm{c}$ are plotted. As stated above, his results and the present ones agree as closely as the curves can be read for the case shown in Figure $6 a$.

Another study which was made with regard to the two-dimensional Luneberg lens was that of gradually stepping the line source away from the edge of the cylinder. The results of this investigation are exhibited in Figures 7,8 and 9 . In these figures $R$ is the distance of the line source from the center of the cylinder, while in each case $k a=8$. None of these results have been reported before.

The final numerical investigation concerning the Luneberg lens was that of computing the scattered field when a plane wave is incident. The far field in this case is shown in Figure 10 for three particular magnitudes of the incident propagation vector. It is interesting to note that these curves also conform to what one might expect from ray optical considerations.

In all parts of the Luneberg lens study the electric field was parallel to the axis of the cylinder. 
A

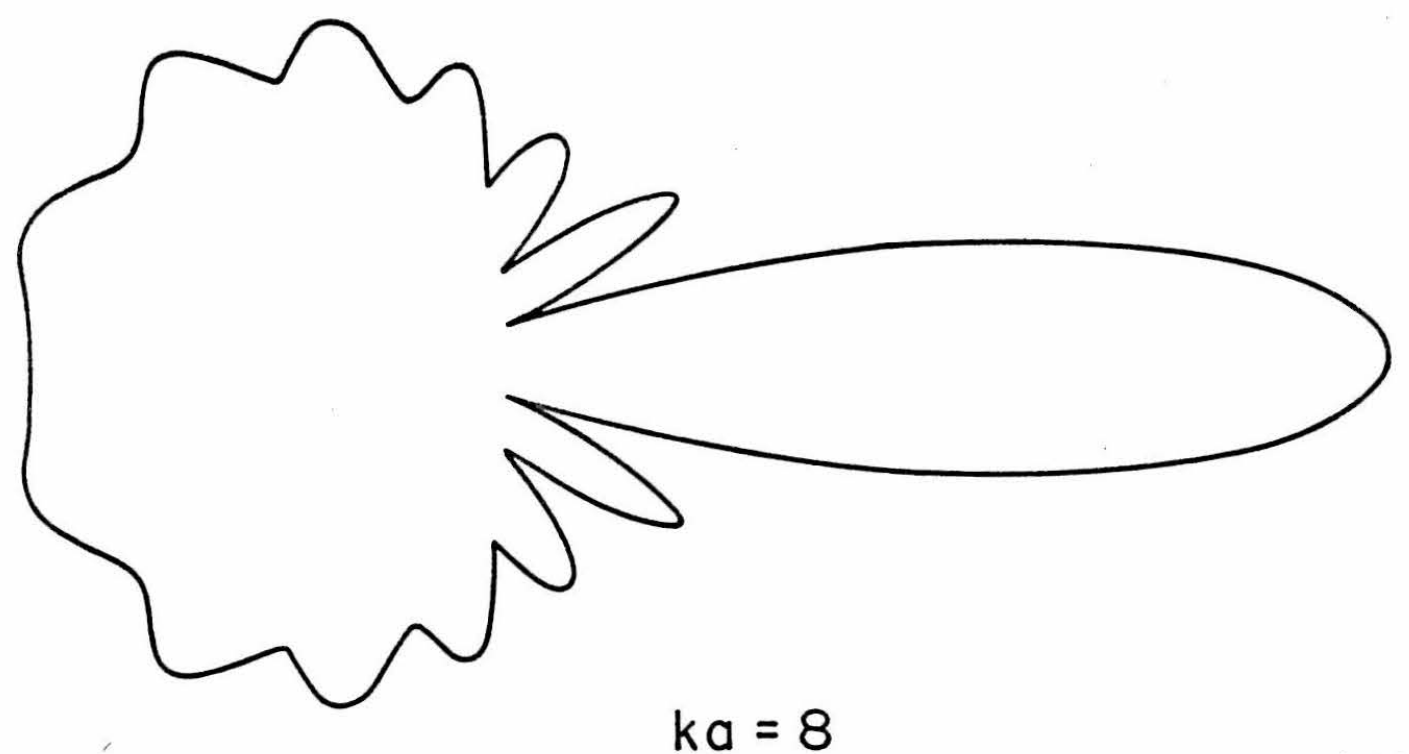

B

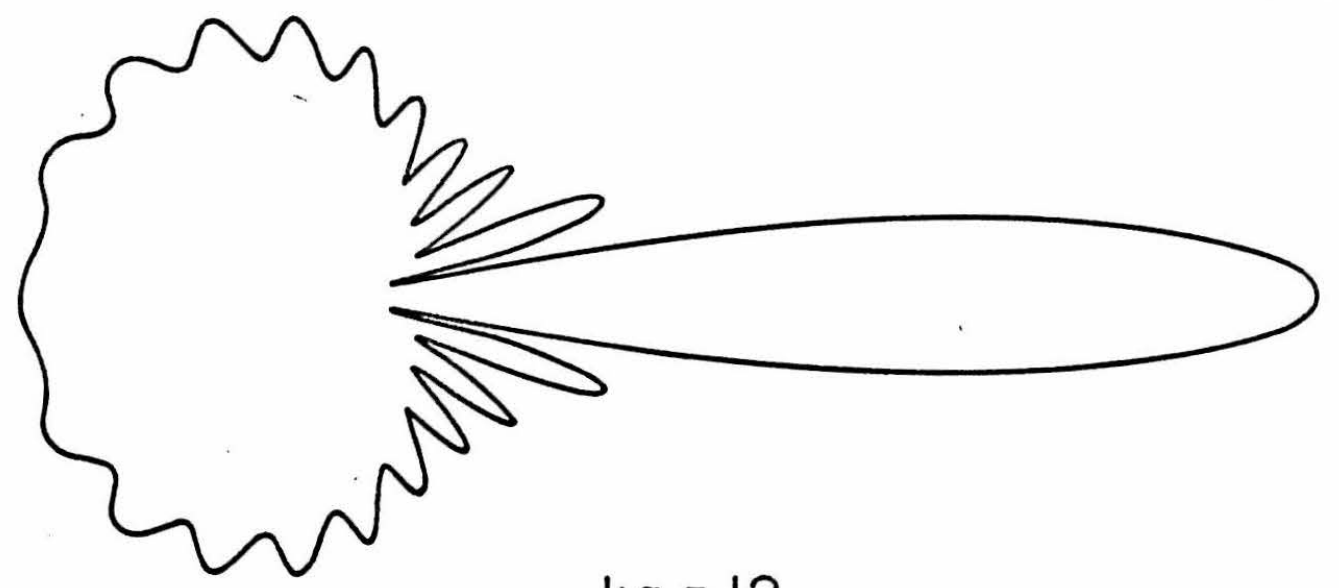

$k a=12$

C

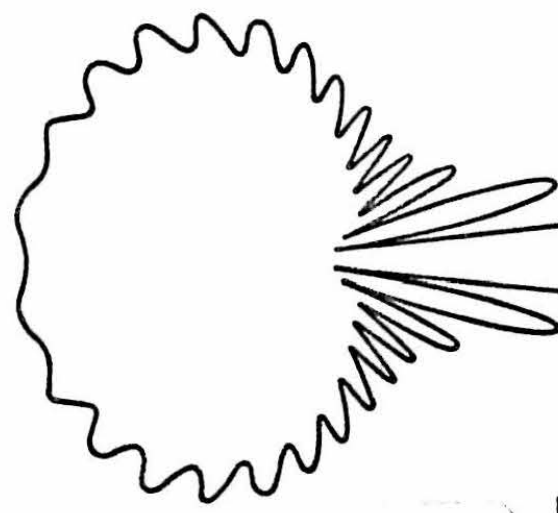

Fig. 6. Far field of a line $k a=16$ source at the edge of a twodimensional Luneberg lens for various frequencies 

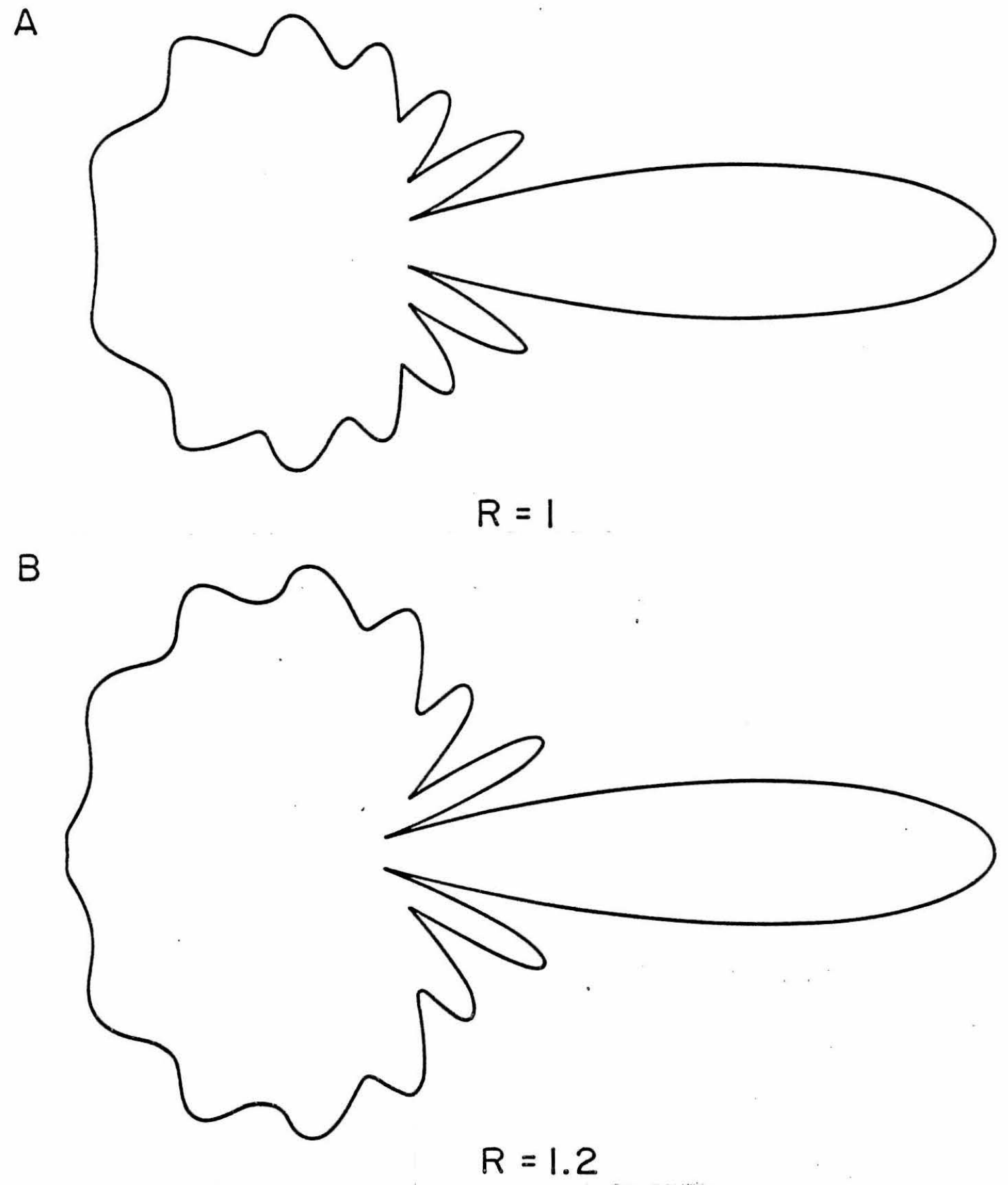

Fig. 7. Far field of a line source at the edge of, and slightly away from a twodimensional Luneberg lens (radius of lens is $1, k$ is 8) 
A
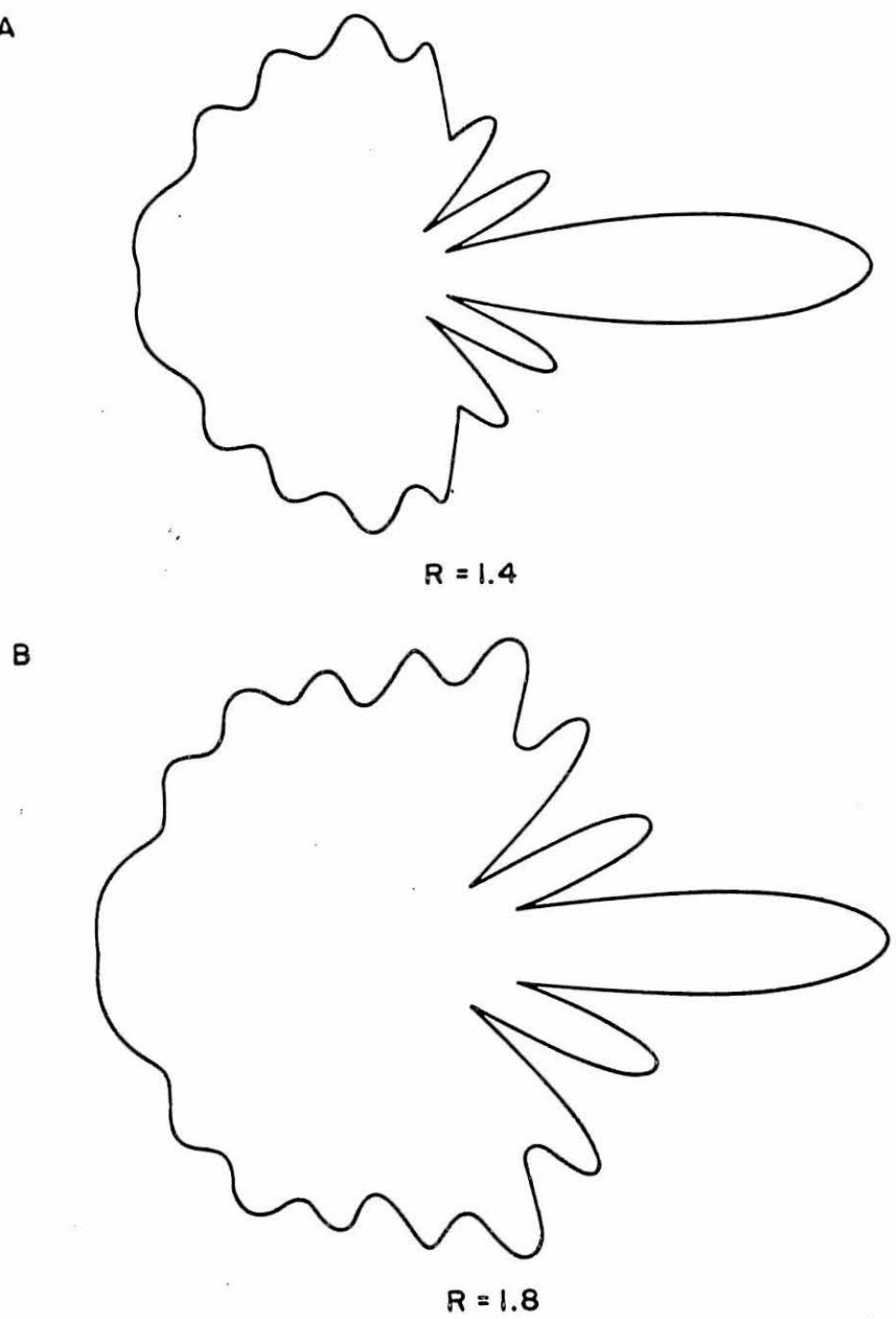

Fig. 8. Far field of a line source at short distances from a Lunebers lens (radius of lens is $1, k$ is 8 ) 
A

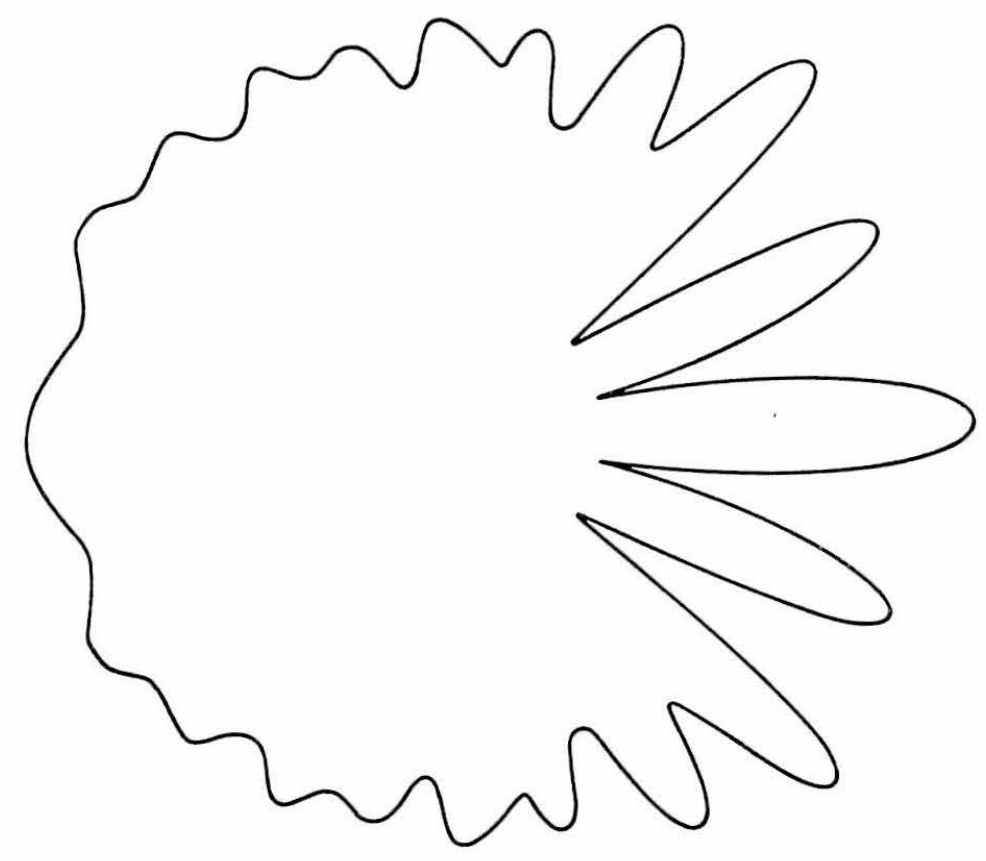

$R=2.5$

B

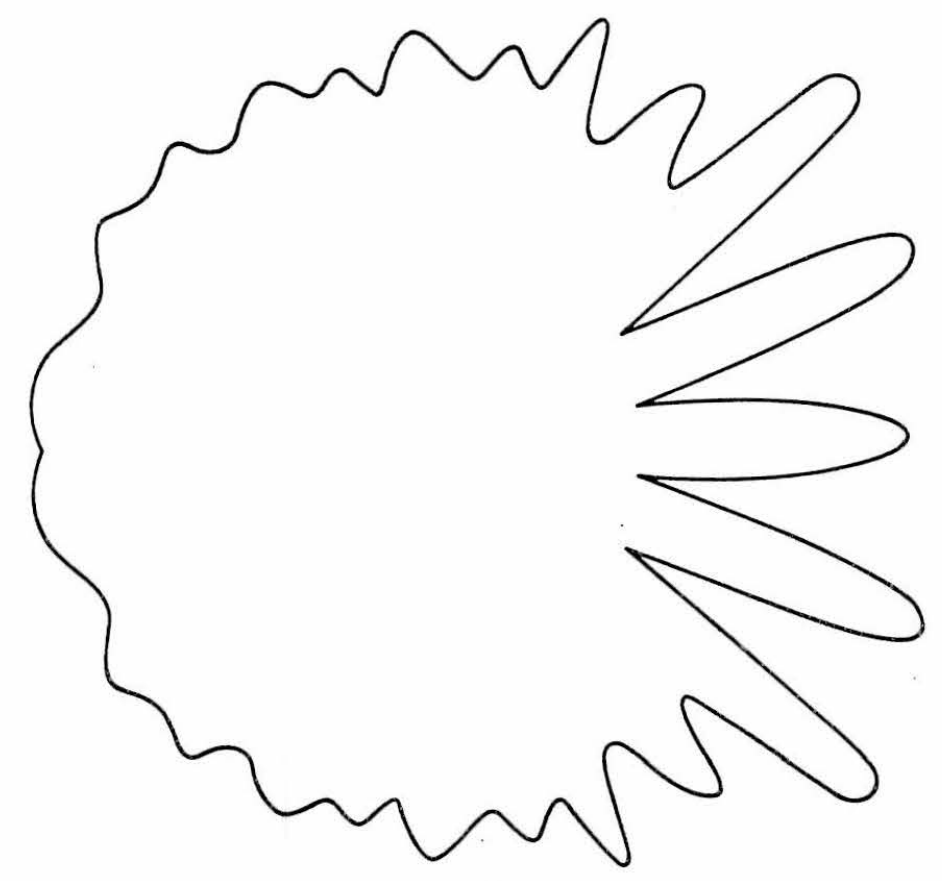

$R=3.0$

Fig. 9. Far field of a line

source at a distance. $R$ from the center of a two-dimensional

Luneberg lens (radius of lens is

$1, \mathrm{k}$ is 8 ) 
A

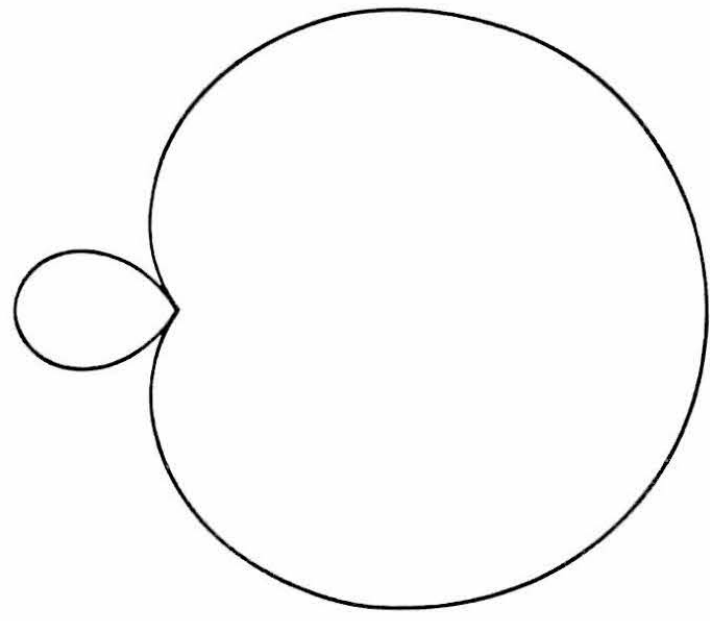

$k a=4$

B

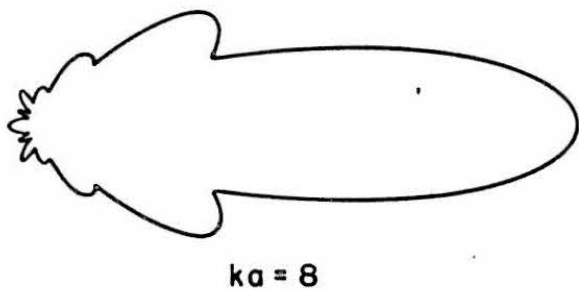

C

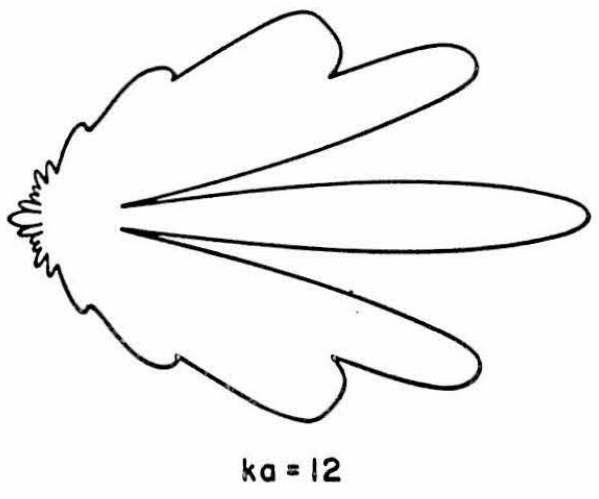

Fig. 10. Scattering patterns of a two-dimensional Luneberg lens at various frequencies 
There are two other types of inhomogeneous cylinders which were examined using the method of this report. The first of these, studied mainly because of the availability of results to check the computer code for the case when the scattering cylinder has a complex dielectric constant, is that reported in reference (II). That is to say, a permittivity variation of the form

$$
\varepsilon(\rho / \lambda)=\left\{\sum_{n=0}^{3} \varepsilon_{2 n}(\rho / \lambda)^{2 n}\right\} \varepsilon_{0}, \quad \varepsilon(a / \lambda)=\varepsilon_{0}
$$

where the $\varepsilon_{2 n}$ are complex constants, is assumed. Part of the results of this investigation are shown in Figure 11 . Figure 11 is a plot of the log of the backscatter, where

$$
R M \equiv \pi^{2} \frac{\rho^{2}}{\lambda^{2}} \cdot\left|\frac{E(\pi)}{E^{\text {inc }}}\right|^{2}
$$

versus the plasma frequency, normalized to the frequency of the incident wave

$$
\overrightarrow{f_{p}^{2}}=\frac{f_{p}^{2}}{f^{2}}
$$

while $\bar{v}$ is the collision frequency relative to the frequency of the incident wave. These parameters are chosen in agreement with the notation of Reference 1l. Figure 11 represents the results for the special case when $\varepsilon_{2 n}=0, n>0$, and it is in agreement with the results of reference (11), (Figure 2), except for certain minor discrepancies near $\bar{f}_{p}^{2}=1$. 


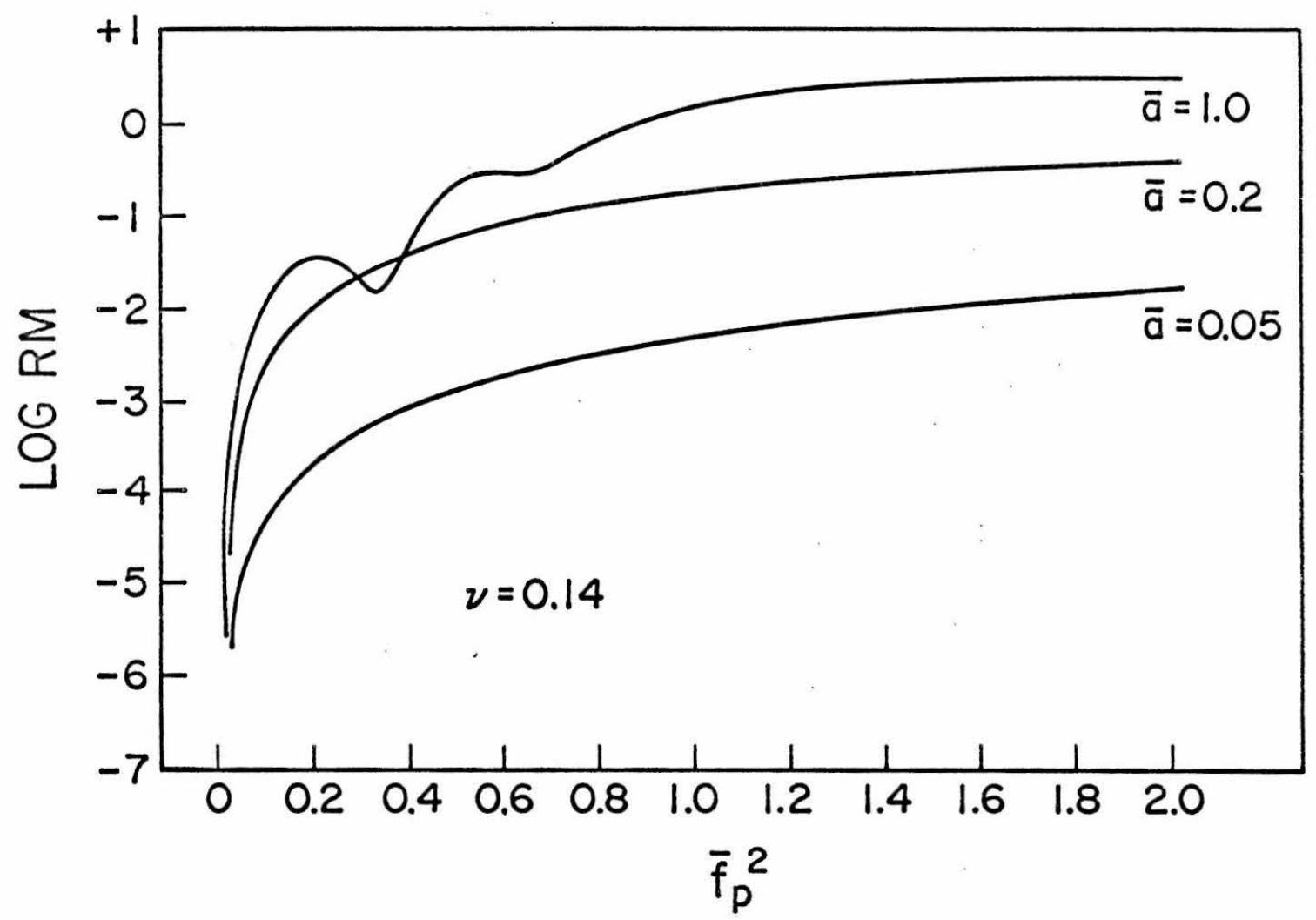

Fig. 11. Back scatter of a homogeneous plasma cylinder under a "collision frequency" approximation 
The last type of cylinder studied was that of an inhomogeneous plasma with the electron density profiles shown in Figure 12. These profiles are of interest since they are semi-empirical results showing the approximate density profiles in the trail behind a rocket, at certain distances behind the rocket. The curves were obtained from the Douglas Aircraft Company.

In Figure 13 the field scattered from profile A is plotted for the case when the incident wave number $(k)$ is six. Both electric and magnetic polarizations are included. Figure 14 consists of similar plots for a different magnitude of the incident wave number. Figures 15 and 16 contain the results for profile B. 


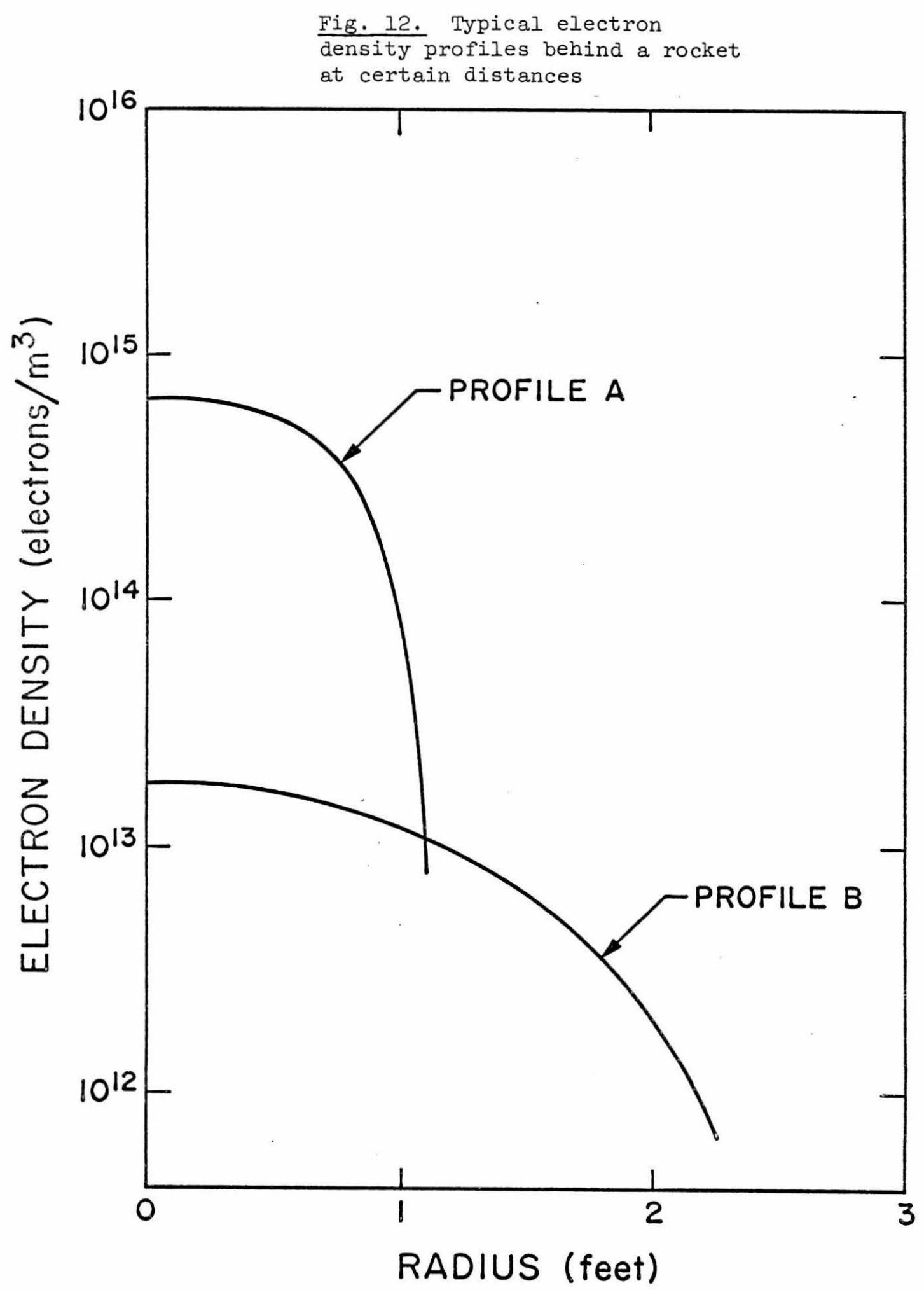


A

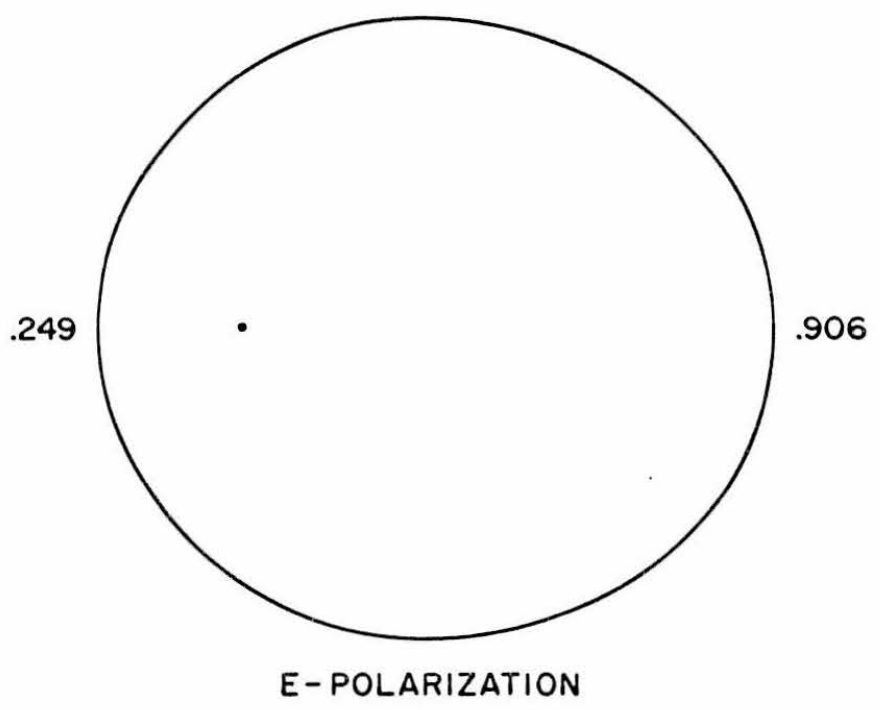

B

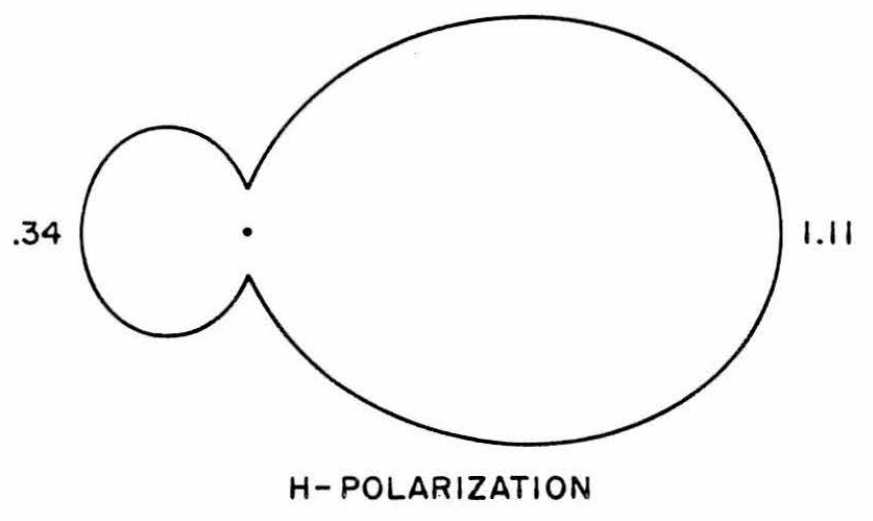

PROFILE A, $k=6, f_{c} / f=.14$

Fig. 13. Differential Scattering widths for Profile A, Fig. 12. 
A

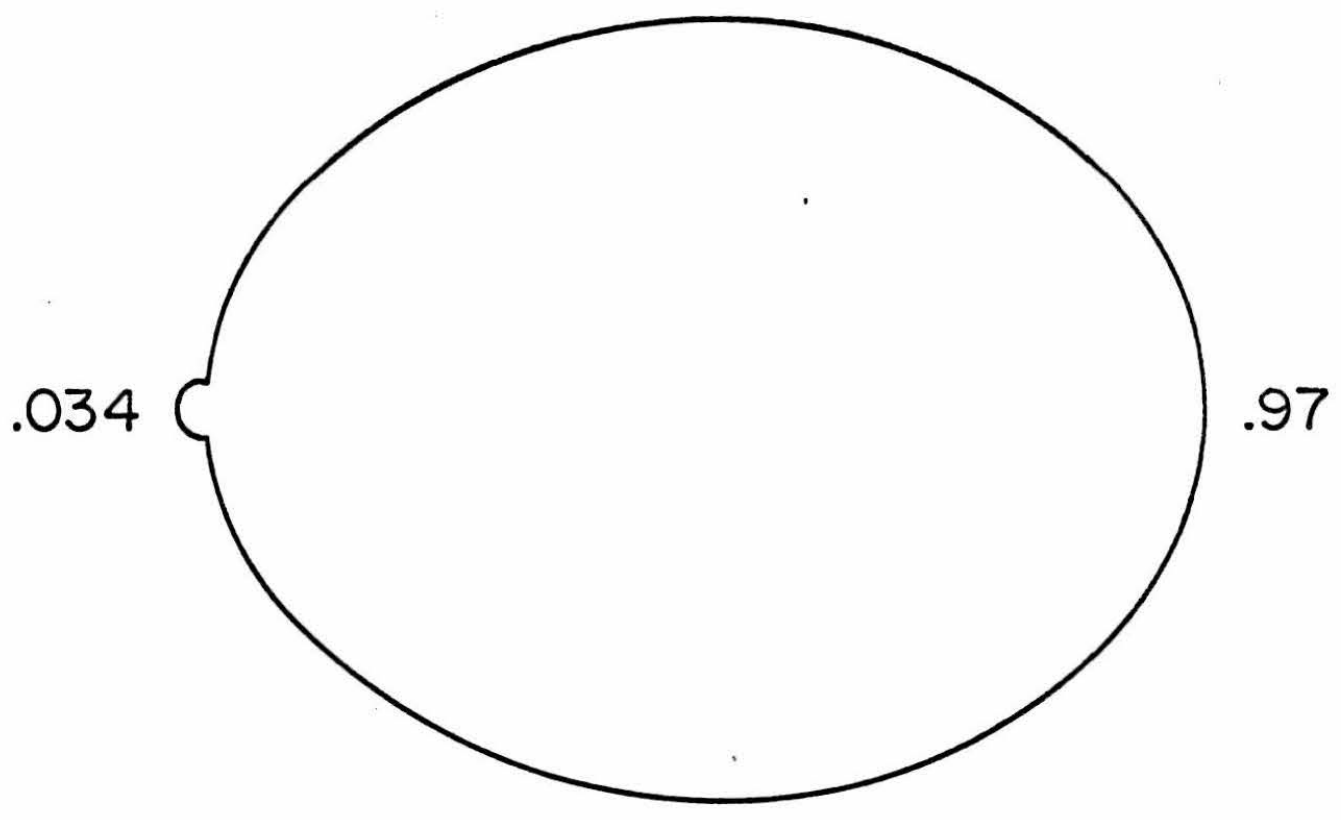

E-POLARIZATION

B

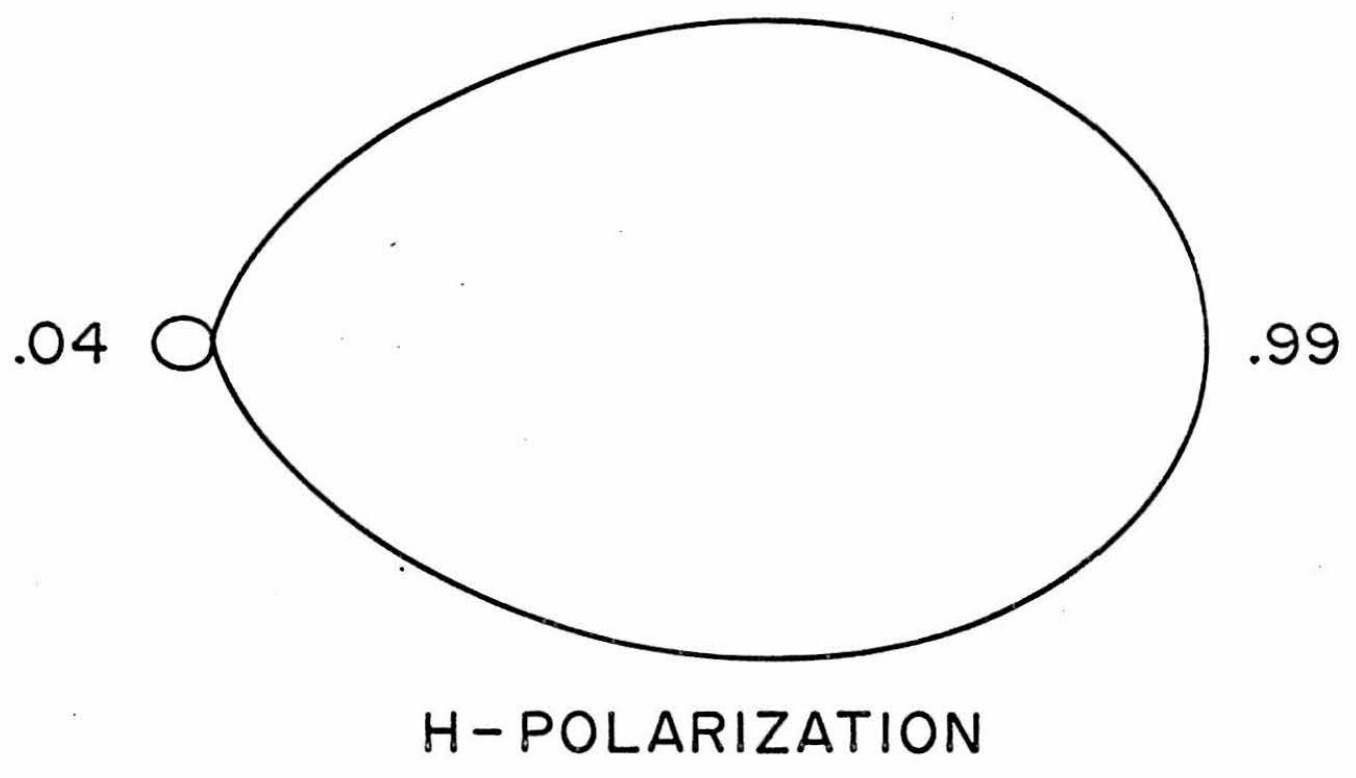

PROFILE $A, k=10, f_{c} / f=.14$

Fig. 14. Differential scatter-

ing widths for Profile A, Fig.12 
A.

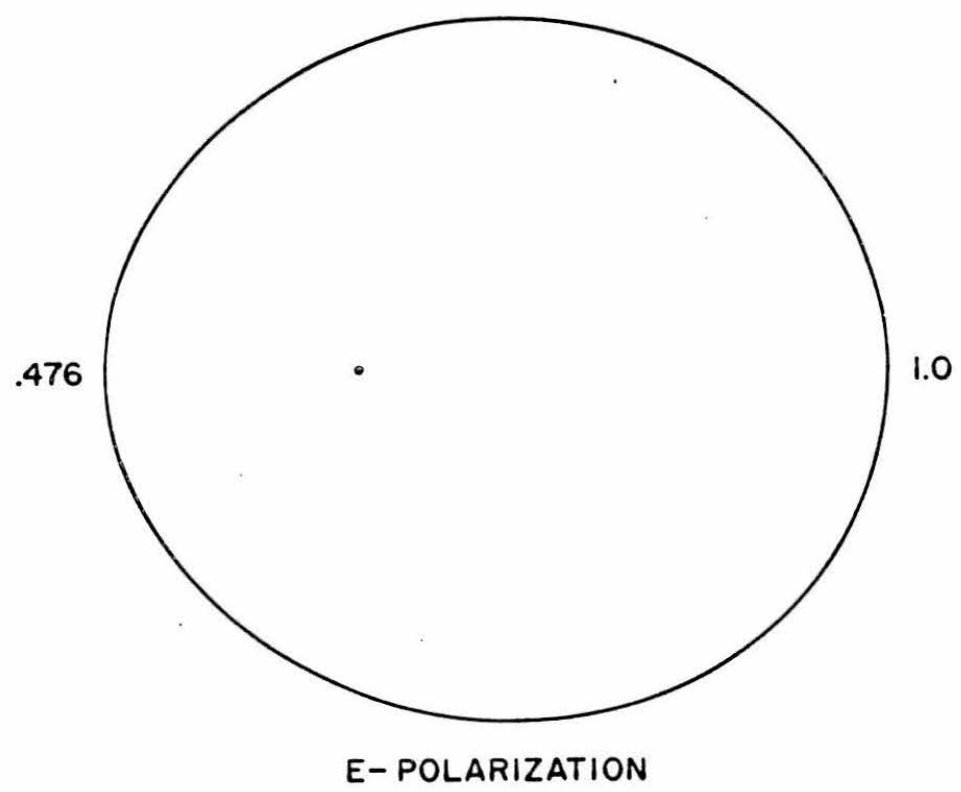

B

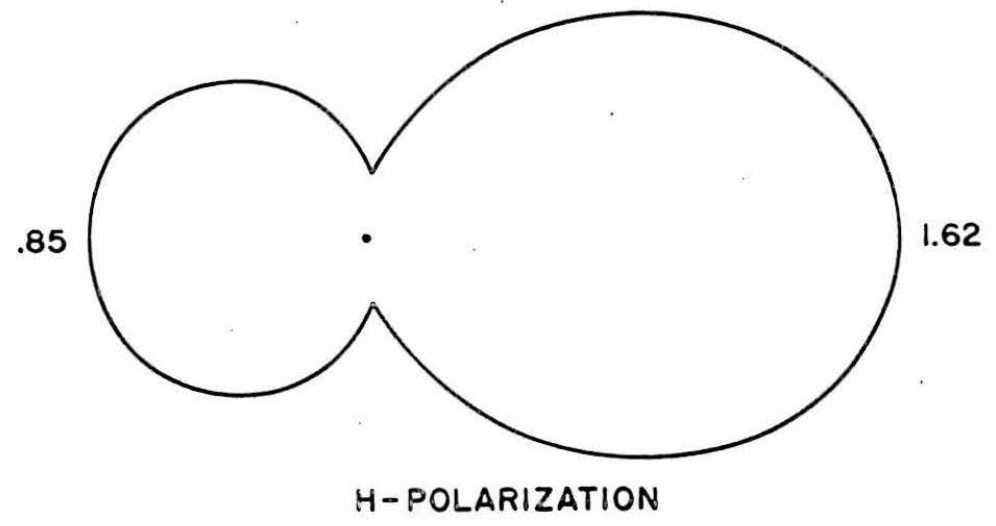

PROFILE B, $k=2, f / f=.14$

Fig. 15. Differential scattering widths for Profile B, Fig. 12. 
A

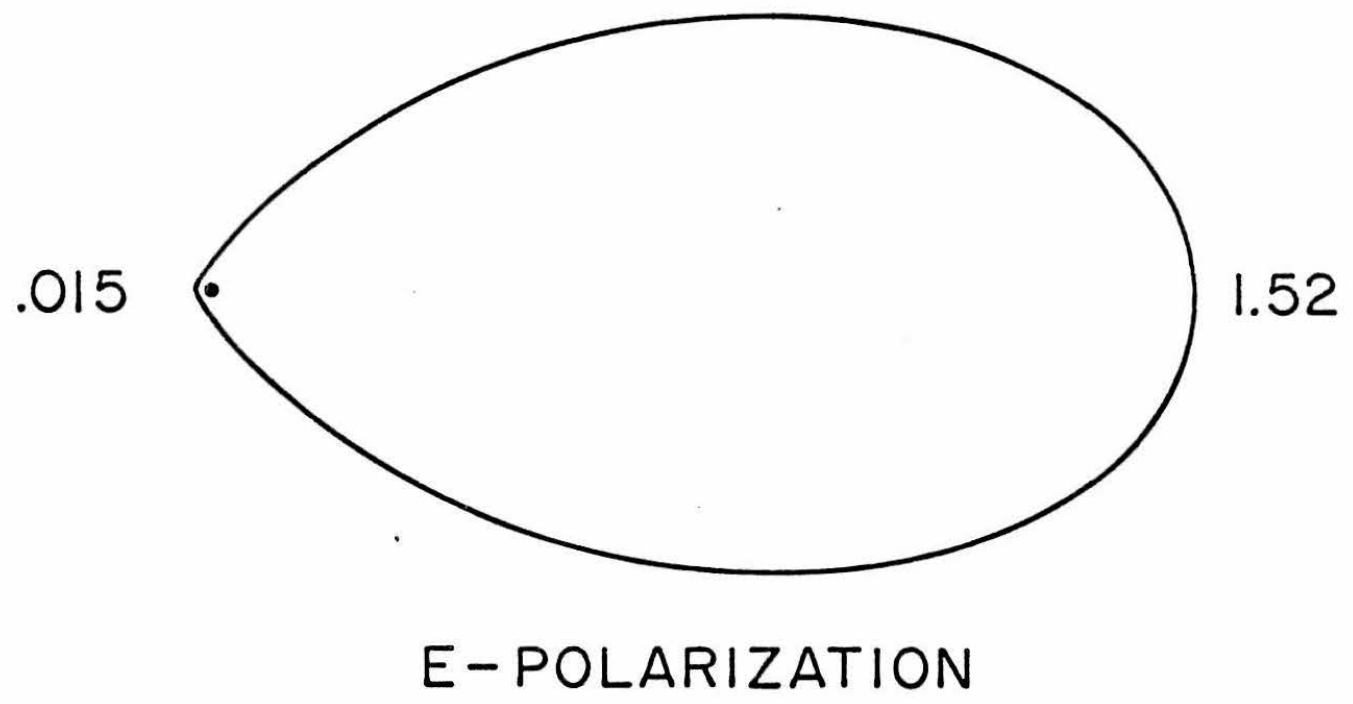

B

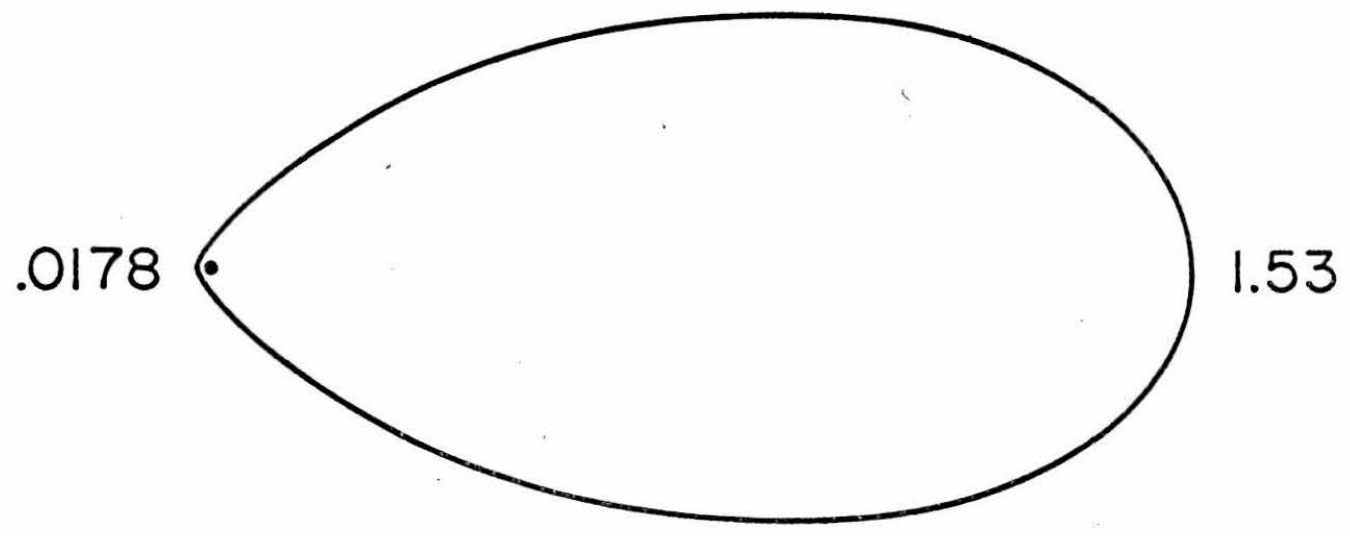

H-POLARIZATION

PROFILE $B, k=6, f_{c} / f=.14$

Fig. 16. Differential scattering

widths for Profile B, Fig. 12 


\section{Section 6}

CONCLUDING REMARKS

As mentioned before, the main point of this work is the practical calculational method that is offered. The method is particularly effective when it is necessary to compute certain data (the far field, for example) for a whole class of problems. That is to say for instance, if one wanted to compute the field scattered from a large group of homogeneous cylinders which differed only in their radius, the method would be the ideal tool for the job. However, even if one only desires information about a particular inhomogeneous cylinder, the method still has advantages. One of these advantages is that the time for obtaining an accurate numerical solution of a'linear second order differential equation has been eliminated by reformulating the problem. In the reformulation there arises a certain nonlinear first order equation. As far as numerical solutions on a digital computer are concerned, the linearity or nonlinearity of the equation is immaterial and there is a definite saving of time from the reduction of the order of the equation.

The advantages the method offered in this report has over those methods which have been put forward in the past are discussed in more detail in the fourth section. Here it should be said, however, that the main differences between the present approach and what might be called the "multi-layer" approach are those of numerical accuracy and a confidence that the limiting form of the numerical solution is truly the quantity which is to be calculated. 
There is one other advantage of the present method over the multilayer approach which should be mentioned; that is, with the analytical forms of the equations inherent in the equation-of-change approach, certain transformations which simplify the calculations for certain parameter values are perhaps a little more transparent. This can be seen, for example, in the approximations leading to equation 3.24 . From the strictly numerical thin-shell approach, these simplifications might never have presented themselves.

Although the main line of this report was the development of a calculational method, there were certain other interesting points that have occurred during the course of the analysis. It is perhaps desirable to give a brief summary of some of them in order that they may not be completely forgotten.

One minor point of this nature is the discussion in the second section of the various terminologies that have been used in discussing problems of this type and the fact that words such as "invariant imbedding", "equation-of-change", etc. have sometimes overlapping but not equivalent meanings.

Another point, more important perhaps, is the various forms that the equation-of-change can take under certain transformations. Some of these (10) are more suitable than others for particular applications. Their suitability depends upon the rate-of-change of the medium parameters, the form in which the boundary conditions are known, etc。

Within this group of subjects it is worth while to point out the compact demonstration of the relation between the transmission 
coefficients in each direction for a one-dimensiona inhomogeneity. This relation is given by equation 2.43 for a general (i.e., conductivity and permeability as well as permittivity) variation of the medium parameters.

Finally, there is the interesting case of a constant ratio of permeability to permittivity to be examined. In the cylindrical geometry this leads to a scattering pattern which is independent of the polarization of the incident plane wave, while in the spherical geometry this variation leads to a backscattering cross-section of zero (as in the one-dimensional case).

There are, of course, certain other points that could be mentioned, but a line must be drawn somewhere. These other points will become evident on a close reading of Sections 2 and 3 .

This report would be incomplete without a discussion of possible extensions of its method. The first of these extensions could be to other geometries. The case of an elliptical, cylindrical inhomogeneity looks straightforward, while that of an ellipsoidal inhomogeneity may be possible for certain of the incident modes. A second area of extension is that of attempting to get a rate-of-change equation for the whole scattered field at once for some three-dimensional problem. An example of this nature which may or may not be possible, is that of trying to get the change in the field of a dipole above a stratified half-space as the position of the dipole recedes from the surface of the half space.

A third, and most important way in which the methods presented here could be extended is in a discussion of more general 
inhomogeneities than those described by a simple statement of dielectric constant as a function of position. An interesting case of this sort is contained in a recent paper (8) discussing the reflection of electromagnetic waves from a slab with a laminar velocity profile. Perhaps a method of treating a turbulent half-space, or slab, could be developed with the invariant-imbedding approach. 
Appendix

COMPUTER CODE

This appendix is a description of a typical computer code for calculating scattered fields using the method of Section 3 . In particular, the following code was used in the computation of most of the data displayed in the fifth section.

For convenience we divide the following brief descriptions into sections based on the programs into which the code itself is divided. CYL

This is the main program. It reads in and writes out the data and juggles the various subroutines. It consecutively

1. Sets up commons

2. Reads: exterior region parameters ( $k_{\min }, k_{\max }$, number of $\left.\mathrm{k}^{\prime} \mathrm{s}\right)$; desired field angle data (min. and max. $\varnothing$, number of $\emptyset^{\prime} s$ ); data concerning number of modes, number of discontinuities, number of integration points per region, number of additional properties which will be fed in, and the initial radius data. positions of discontinuities additional properties used for miscellaneous calculations type of source, polarization, radius of source, if a line, real and imaginary parts of source strength, if a line.

3. Sets up loop of varying $k_{c}$

4. Calls subroutine COEF (description later)

5. Determines starting unknowns 
6. Calls CONT up to first discontinuity in the medium

7. Calls PARA for $n$th and $n+1$ th regions and then HOP

8. Determines whether the last region has been reached, and if not, calls CONT, etc.

9. Calls REF and SUM

10. Writes out titles, some identifying data, and the far field for various angles

11. Sets formats.

The computations are based on the scheme embodied in equations

3.28 and 3.29. The subroutines mentioned in the above description are, in order:

$\underline{\mathrm{COEF}}$

This subroutine calculates the modal coefficients of the incident wave in two often-used cases, namely

(a) plane wave

$$
\begin{aligned}
\mathrm{COR}+\mathrm{COI} & =1+i 0 \\
\mathrm{C}_{\mathrm{n}} & =(-1)^{\mathrm{n} / 2} \mathrm{n} \text { even } \\
& =(\mathrm{n} /|\mathrm{n}|)(-1)^{(\mathrm{n}-1) / 2} \mathrm{n} \text { odd }
\end{aligned}
$$

(b) line source at $\rho_{0}, \pi$ of strength $R+i I$

$$
\begin{aligned}
& C_{0}=(R+i I)\left(J_{0}\left(k_{0} \rho_{0}\right)+i Y_{0}\left(k_{I} \rho_{0}\right)\right) \\
& C_{n}=(R+i I)\left(J_{n}\left(k_{0} \rho_{0}\right)+i Y_{n}\left(k_{0} \rho_{0}\right)\right)(-I)^{n}
\end{aligned}
$$

BEGIN

This routine gets the program off the ground at small radius by using the small argument expansions for the impedance-admittance variable. 


$$
\begin{array}{ll}
\mathrm{Z}_{1}=-\mathrm{k} \rho / 2 & \mathrm{U}(1)=0 \\
\mathrm{Z}_{\mathrm{n}}=\mathrm{k} \rho /(\mathrm{n}-1) & \mathrm{U}(\mathrm{n})=1
\end{array}
$$

Note that the $U$ variable determines whether we are using equation $3.28(U=0)$ or $3.29(U=1)$.

$\underline{\text { PARA }}$

This routine supplies the medium parameters of the particular inhomogeneity under study. In general,

$$
\text { (a) } \frac{\dot{Z}}{Z}=X D+i Y D=\frac{1}{2}\left\{\frac{\dot{\mu}}{\mu}-\frac{\dot{\varepsilon} / \varepsilon+\alpha 2 \dot{\sigma} / \sigma}{1+\alpha^{2}}\right\}+\frac{i \alpha}{2}\left\{\frac{\dot{\varepsilon} / \varepsilon-\dot{\sigma} / \sigma}{1+\alpha^{2}}\right\}
$$

where $\alpha=\sigma / \omega \varepsilon$

$$
\begin{aligned}
& \text { (b) } Z=X+i Y=\frac{Z c}{\sqrt{2 M}}(\sqrt{M+1}+i \sqrt{M-1}) \text { where } M=\sqrt{1+\alpha^{2}} \\
& \text { (c) } k=k_{c} \sqrt{\frac{M}{2}}(\sqrt{M+1}+i \sqrt{M-1} \text {. }
\end{aligned}
$$

In the particular case of the lossless, cylindrical Luneberg lens, these reduce to

$$
\frac{\dot{Z}}{Z}=\frac{\rho}{\rho_{c}^{2}-\rho^{2}} \quad k=k \rho \sqrt{2-\frac{\rho^{2}}{\rho_{c}^{2}}} \quad z=\frac{z_{c}}{\sqrt{2-\rho^{2} / \rho_{c}^{2}}}
$$

For H-polarization, because of the set-up of the program

$$
\begin{aligned}
\dot{\mathrm{Z}} / \mathrm{Z} & \rightarrow-\dot{\mathrm{Z}} / \mathrm{Z} \\
\mathrm{Z} & \rightarrow \mathrm{I} / \mathrm{Z}
\end{aligned}
$$


CONT

This routine integrates numerically using Heun's method (12) equation 3.28 or 3.29 , within regions in which the medium is continuous.

$\underline{\text { HOP }}$

This is a simple routine which, from the impedances of the plus and minus regions and the impedance-admittance in the minus region, computes the impedance-admittance in the plus region according to formula 3.30 .

$\underline{\mathrm{REF}}$

This subroutine calculates the reflection coefficients from the impedance-admittance $\phi_{n}$, using the formula

$$
R_{n}=\frac{\phi_{n} J_{n}-J_{n}^{\prime}}{H_{n}^{(I)}-\phi_{n} H_{n}^{(I)}}
$$

or

$$
R_{n}=\frac{J_{n}-\phi_{n} J_{n}^{\prime}}{\phi_{n} H_{n}^{(I) '}-H_{n}^{(I)}}
$$

depending upon whether the indicator variable $U(n)$ is zero or one, respectively. We use the formulas:

$$
\begin{aligned}
J_{n}^{\prime} & =\frac{1}{2}\left(J_{n-1}-J_{n+1}\right) \\
H_{n}^{(I)^{\prime}} & =\frac{1}{2}\left(H_{n-1}^{(1)}-H_{n+1}^{(I)}\right)
\end{aligned}
$$

and so obtain for the two cases, if $\emptyset_{n}=R+i X$ 
$\mathrm{U}(\mathrm{n})=0$

$$
\begin{aligned}
& R_{0}=\frac{R J_{0}+J_{I}-i X J_{0}}{-J_{I}-i Y_{I}-(R+i X)\left(J_{0}+i Y_{0}\right)} \\
& R_{n}=\frac{\left(R J_{n}-\frac{J_{n-1}-J_{n+I}}{2}\right)+i X J_{n}}{\left(\frac{J_{n-1}-J_{n+1}}{2}\right)+i\left(\frac{Y_{n-1}-Y_{n+I}}{2}\right)-(R+i X)\left(J_{n-I} Y_{n}\right)}
\end{aligned}
$$

$$
\begin{aligned}
U(n)=1 \quad R_{0} & =\frac{J_{0}+(R+i X) J_{1}}{-(R+i X)\left(J_{1}+i Y_{1}\right)-J_{0}-i Y_{0}} \\
R_{n} & =\frac{J_{n}-(R+i X)\left(\frac{J_{n-1}-J_{n+1}}{2}\right)}{(R+i X)\left(\frac{J_{n-1}-J_{n+1}}{2}+i \frac{Y_{n-1}-Y_{n+1}}{2}\right)-J_{n}-i Y_{n}}
\end{aligned}
$$

SUM

This subroutine calculates the far field as a function of angle, using the reflection coefficients of the individual modes and the modal coefficients of the incident wave, i.e., we do the sum

$$
E, H=A_{0} R_{0}+\sum_{n-1}^{\infty}(-i)^{n} R_{n}\left\{A_{n} e^{i n \phi}+(-1)^{n} A_{m} e^{-i n \phi}\right\}
$$

In addition to the above codes, use is made of a CIT library subroutine for calculating Bessel functions. 
REFERENCES

1. Jasik, H., "The Electromagnetic Theory of the Luneberg Lens", Air Force Cambridge Research Center AFCRC-TR-54-121; November 1954.

2. Ambarzumian, V. A., "Diffuse Reflection of Light by a Foggy Medium" Compt. Rend. Acad. Sci. U.R.S.S. 38, No. 8, 1943, pp. 229-232.

3. Chandrasekhar, S., Radiative Transfer, Dover Publications, New York, 1960.

4. Bellman, R., and R. Kalaba, "On the Principle of Invariant Imbedding and Propagation through Inhomogeneous Media", Proc. Nat. Acad. Sci USA 42, 1956, pp. 629-632.

5. Bellman, R., and R. Kalaba, "Invariant Imbedding, Wave Propagation and the W.K.B. Approximation", Proc. Nat. Acad. Sci. USA $4 \underline{4}$, 1958, pp. 317-319.

6. Papas, C. H., "Plane Inhomogeneous Dielectric Slab", Caltech Antenna Laboratory Note, March 1954.

7. Stratton, J.A., Electromagnetic Theory, McGraw-Hill, New York and London, 1941, p. 564.

8. Wagner, R. J. and P. J. Lynch, "Theorem on Electromagnetic Backscatter", Phys. Rev. 131, No. I, 1963, pp. 21-23.

9. Kritikos, H. N., K.S.H. Lee, and C. H. Papas, "Electromagnetic Reflectivity of Non-Uniform Jet Streams", Caltech Antenna Laboratory Report, August 1966.

10. Pierce, J。, Bell Syst. Tech. Journal 22, 1943, pp. 263-265.

11. De Ridder, C. M. and S. Edelberg, "Scattering from Plasma Cylinders with Radial Variations in Electron Density", in Electromagnetic Aspects of Hypersonic Flight, Sparton Books, 1964, pp. 286 ff.

12. Todd, J., Survey of Numerical Analysis, McGraw Hill, New York, 1962, pp. 79-80。 
13. Suokes, G. G., "On the Intensity of the Light Reflected from or Transmitted through a Pile of Plates", Proc. Roy. Soc. 11, 1862, pp. 545-556.

14. Brekhovskikh, L., Waves in Layered Media, Academic Press, New York and London, 1960.

15. Budden, K. G., Radio Waves in the Ionosphere, Cambridge University Press, Cambridge, 1961.

16. Wait, J. R., Electromagnetic Waves in Stratified Media, The Macmillan Company, New York, 1962, pp. 100-105.

17. Luneberg, R. K., "The Propagation of Electromagnetic Plane Waves in Plane Parallel Layers", Research report Nos. 172-173, New York University, 1947.

18. Barrar, R. B. and R. M. Redheffer, "On Nonuniform Dielectric Media", IRE Trans. on Antennas and Propagation, $\underline{A P-3}, 1955$, pp. 101-107.

19. Bellman, R. and R. Kalaba, "On the Principle of Invariant Imbedding and Diffuse Reflection from Cylindrical Regions", Proc. Nat. Acad. Sci. USA 43 , 1957, pp. 517-520.

20. Bellman, R. and R. Kalaba, "Functional Equations, Wave Propagation and Invariant Imbedding", J. Math. Mech. 8, 1959, pp. 683-704.

21. Bellman, R. and R. Kalaba, "Invariant Imbedding and Wave Propagation in Stochastic Media" in Electromagnetic Wave Propagation, M. Desirant and J. I. Michiels (Eds.), Academic Press, New York, 1960, pp. 243-252.

22. Bellman, R., R. Kalaba and G. M. Wing, "Invariant Imbedding Conservation Relations, and Non-Linear Equations with Two-Point Boundary Values", Proc. Nat. Acad. Sci. USA 46, 1960, pp. 1258-1280.

23. Redheffer, R., "Difference and Functional Equations and Transmission Line Theory" in Modern Mathematics for the Engineer, Series II, E.F. Beckenbach (Ed.), McGraw Hill, New York, 1961, pp. 282-337. 
24. Preisendorfer, R., "Invariant Imbedding Relation for the Principles of Invariance", Proc. Nat. Acad. Sci. USA 44, 1958, pp. 320-323.

25. Preisendorfer, R., "Functional Relations for the R and $T$ Operation on Plane-Parallel Media", Proc. Nat. Acad. Sci. USA 44, 1958, pp. 323-328. 